\title{
Coping and Depression in Old Age: A Literature Review
}

\author{
Guro Hanevold Bjørkløfa Knut Engedal $^{\text {b-d }}$ Geir Selbæk ${ }^{d, e}$ \\ Siren Eriksen Kouwenhoven ${ }^{c, f}$ Anne-Sofie Helvik ${ }^{g-i}$ \\ a Department of Psychiatry, Vestre Viken Hospital Trust, Lier, ${ }^{b}$ Norwegian Centre for Dementia \\ Research, Oslo University Hospital, Ullevaal, ' ${ }^{\text {Faculty }}$ of Medicine, University of Oslo, Oslo, ${ }^{\mathrm{d}}$ Centre \\ for Old Age Psychiatric Research, Innlandet Hospital Trust, Ottestad, eAkershus University Hospital, \\ Lørenskog, ${ }^{f}$ Buskerud University College, Drammen, Kongsberg, 9 Department of Public Health and \\ General Practice, Faculty of Medicine, Norwegian University of Science and Technology (NTNU), \\ and ' St Olav's University Hospital, Trondheim, and 'Innlandet Hospital Trust, Division Tynset, Tynset, \\ Norway
}

\section{Key Words \\ Coping $\cdot$ Depression $\cdot$ Older persons}

\begin{abstract}
Background/Aims: The interest in the relation between coping and depression in older persons is growing, but research on the concepts and instruments of coping in relation to depression among older persons is scarce and systematic reviews are lacking. With this background, we wanted to gain a systematic overview of this field by performing a systematic literature search. Methods: A computer-aided search in MEDLINE, CINAHL, PsycINFO, Embase, PubMed and www.salutogenesis.fi was conducted. We systematically searched for studies including coping and depression among persons 60 years of age and above. The included studies were evaluated according to predefined quality criteria. Results: Seventy-five studies, 38 clinical and 37 community settings, were included. Of these, 44 were evaluated to be of higher quality. Studies recruiting samples of older persons with a major depressive disorder, moderate or severe cognitive impairment or those who were dependent on care were scarce, thus the research is not representative of such samples. We found a huge variety of instruments assessing resources and strategies of coping (55 inventories). Although we found the relation between resources and strategies of coping and depression to be strong in the majority of studies, i.e. a higher sense of control and internal locus of control, more active strategies and positive religious coping were significantly associated with fewer symptoms of depression both in longitudinal and cross-sectional studies in clinical and community settings. Conclusion: Resources and strategies of coping are significantly associated with depressive symptoms in late life, but more research to systematize the field of coping and to validate the instruments of resources and strategies of coping in older populations is required, especially among older persons suffering from major depression and cognitive decline.
\end{abstract}


Bjørkløf et al.: Coping and Depression in Old Age: A Literature Review

\section{Introduction}

According to a review article by Rosenvinge and Rosenvinge [1], 10-19\% of older persons in the general population suffer from symptoms of depression, and $2-4 \%$ suffer from a major depressive disorder. Among older people living in an institution, a systematic review reports a median prevalence of major depression of $10 \%$, and of depressive symptoms in $29 \%$ of the residents $[2,3]$. Another study showed that about $50 \%$ of elderly people in long-term care suffer from depressive symptoms and $20 \%$ from major depression [4]. A further study reported that about $30 \%$ of all referrals to the specialist psychiatric health service are older people suffering from depression [5]. Since older persons constitute the fastest growing part of the population and because depression is among the most common psychiatric disorders in this group, the future cost of depression to the patient, the carer and the health services will be significant [6].

In a review on prognostic factors for depression in older persons the following variables were found to lead to a poor prognosis: older age, chronic somatic comorbidity, more functional limitations, a higher baseline depression level and the locus of control (LOC) being more external than internal [7]. In a systematic review comparing the prognosis for depression in different age groups, remission rates of depression show little difference between middleaged and older persons, but relapse rates appear higher in older persons [8]. Depression in older people is related to increased mortality [9]. This makes it important to further investigate the prognosis for older persons suffering from depression and to include the concept of coping, as this factor is reported to be relevant in the understanding of depression in older persons $[10,11]$.

\section{The Concept of Coping}

The concept of coping was first adopted by psychologists in the 1960s and 1970s and was applied to refer to the struggle of overcoming and managing the stresses of living and adapting [12]. Different theoretical perspectives have defined coping as personality traits where the way a person copes is determined by the kind of person they are [13], and as a process where coping is seen as a situation-specific and flexible state [12]. Situational factors may be the changeability or controllability of a situation, and personality factors may include the aspects of self-confidence, self-efficacy [14] and LOC [15], two central concepts of the theories of personality called control orientation that constitutes parts of a person's total available coping resources [16]. LOC relates to the generalized expectations regarding who or what is responsible for the outcomes. If the person attributes the outcome to luck or powerful others, the belief is labeled external control, and if the relation is attributed to personal effort, the belief is labeled internal control [15, 17]. Self-efficacy refers to the perception that one has the abilities to enact these responses [14], and sense of control (SOC) [18] is understood as the perception of control in a certain situation [16].

According to a transactional perspective on coping, the person and the environment are understood to be in an ongoing reciprocal relationship, where the stressors in life are evaluated in an appraisal process according to the perceived personal resources (i.e. control orientation, self-efficacy) and choices available to the coping person to manage the challenges [19]. Coping, as described by Folkman and Lazarus [19], involves different strategies to alter the stressful situation (i.e. problem-focused coping), as well as efforts to regulate the emotional distress associated with the situation (i.e. emotion-focused coping). The strategies chosen depend both upon situational and individual factors: the coping resources. Other theorists have further developed different concepts of coping related to the coping strategies of Folkman and Lazarus [20,21], like coping actions and coping style [22, 23]. 
Also, Pargament [24] shows that the subjective meaning or orientation of values like religiosity is important for decisions and thus serves as part of the system of coping resources that form the basis of the chosen coping strategies, activities and actions. According to Pargament [24], the concept of religiosity can be studied through the entire process of coping in terms of stressors, appraisal, orientation of values, activities and outcome. Conceptually, processes of coping concerning religiosity have become a specific category of coping called 'religious coping'. Religious coping has been operationalized in different ways, but basically as an instrument to measure either religious beliefs or behavior that serve as coping strategies to help manage emotional distress [25]. Religious coping can be positive, like spiritual supportand positive religious reframing, or negative, i.e. seeing the illness as God's punishment [26]. Antonovsky [27], on the other hand, emphasizes that coping is a resource and is, therefore, seen as a personal capacity that can be used in stressful situations to maintain positive health and achieve well-being. To achieve this positive outcome of coping a certain way of viewing the world is required, a perceived SOC, described as '... a dispositional orientation toward stressors, characterized and operationalized by a view of life as being comprehensible, manageable and meaningful' [28]. A high SOC is found to be associated with good mental health [29]. Hence, the concept of coping reflects different parts and perspectives of the coping process. A critical event or stressor may be experienced as a minor or major life event depending on the available internal and external resources, and has to be understood in terms of the subjective meaning and orientation of values. Resources of coping determine outcome, after being mediated by different coping strategies and activities [24].

The growing interest in coping among older persons has stimulated research in the field, but the body of research on coping in relation to depression in old age is scarce. A systematic review would contribute to a better understanding of the field today and may contribute to meeting a growing interest from personnel in both the specialist and primary health care service who serve depressed older persons. On this basis we conducted a systematic computerbased literature review, including studies where the participants had a mean age of 60 years or more, and where different concepts of coping were studied in relation to depression.

\section{Method}

\section{Selection of Studies}

Two researchers (S.E.K./G.H.B.) and a librarian (L.M.W.) conducted systematic, computer-aided searches in MEDLINE, PsycINFO, Embase and CINAHL (last search 11.07.2012). The terms used for searching the databases were 'aged', 'aged, 80 and over', 'gerontology, aged (attitudes toward)', 'aging', 'geriatric psychiatry', 'geriatric psychotherapy', 'geriatric patient', 'elderly', 'elder care', 'depression', 'depressive disorder', 'depressive disorder, major', 'depression reactive', 'affective disorder', 'psychotic, endogenous depression', 'long-term depression', 'organic depression', 'reactive depression', 'coping', 'ways of coping questionnaire', 'locus of control', 'coping behavior', 'internal external LOC', 'exp. coping behavior', 'sense of coherence' and 'SOC'. The MeSH terms and CINAHL headings were limited to major concepts (focused), except the CINAHL heading 'internal external LOC', which became an 'exploded' search. The keywords, CINAHL headings and MeSH terms were combined in different ways to yield maximum results. This paper reviews published research studies on coping in relation to depression in older persons, focusing on:

(i) Identifying which categories of coping have been studied

(ii) The study design

(iii) The characteristics of the sample

(iv) The aim of each study

(v) Identifying which generic measures of coping have been used

(vi) The primary findings of the studies regarding the relationship between coping and depression in older persons

Papers were included in the review if the following criteria were met:

- Mean age $\geq 60$ years 
Bjørkløf et al.: Coping and Depression in Old Age: A Literature Review

- A quantitative design

- Instrument used to assess depression was exclusive to this purpose

- At least one generic measure of coping was used

- An assessment of the relationship between coping and depression was performed

- The study was published in a journal and appeared in the English language

Papers were excluded from the review if:

- They were theoretical, qualitative or review articles or comments on studies

- They were disseminations

In total 1,727 hits were screened for potentially relevant papers among titles and abstracts; 164 articles were then retrieved for full-text evaluation of the inclusion criteria by two of the authors (A.-S.H./G.H.B.) Reference lists were checked to retrieve relevant publications which had not been identified by the computeraided search. Potentially relevant articles retrieved from disseminations were included. Finally, 66 articles were evaluated by two researchers (A.-S.H./G.H.B.) as fulfilling the inclusion criteria and were further analyzed and their relevant data extracted in the form of tables. In addition, a search of the database www. salutogenesis.fi was conducted (G.H.B.; 30.06.2012) and 41 potentially relevant articles were retrieved. Of these, 9 articles met the inclusion criteria. Thus, 75 articles from five databases were evaluated as meeting the criteria for inclusion in this review.

\section{Quality Assessment}

The methodological quality of each of the studies was assessed by two of the authors (G.H.B./A.-S.H.), based on theoretical considerations and methodological aspects and according to a checklist of predefined criteria as described by Licht-Strunk et al. [7] and Oxman [30]. The list contains 7 quality criteria. A study receives 1 point for each of the following criteria: (i) being a longitudinal study, (ii) containing information about the setting, (iii) including more than 100 participants and (iv) applying a definition of coping with reference to the literature; 2 points are given for each of the following criteria: (v) information about the diagnosis of depression according to criteria in the Diagnostic and Statistical Manual of Mental disorders (DSM) or the International Classification of Diseases (ICD), (vi) use of well-established measures of depression and (vii) use of well-established measures of coping (table 1). We chose to give these 3 latter criteria 2 points because valid and reliable information about the assessment of coping and depression in the studies was regarded to be of outmost importance to the evaluation of a relation between coping and depression, which is the main focus of this review. A total score was calculated by summing the number of positively scored criteria (range $0-10)$. We chose to consider a study as 'high quality' when it scored more than 5 points $(\geq 60 \%$ of the maximum attainable score of 10) and 'low quality' when it scored 5 points or less [7].

\section{Results}

\section{Samples of Older Persons}

Of the 75 studies in total, 38 were clinically based and 37 were community based. Of the clinically based studies, 26 were conducted in hospitals or in GP practices, 6 in psychogeriatric clinics, 4 in nursing homes and 2 studies were from memory clinics. The clinical studies included patients with a variety of disorders such as depression in 12 studies, somatic disorders in general (not specified) in 11, different cardiac disorders in 8, cognitive impairment in 2, Parkinson's disease in 2, cancer in 2 and pain disorders in 1 study (tables 2, 3).

\section{Quality of the Studies}

In total, 24 studies had a longitudinal and 51 had a cross-sectional design (tables 2, 3). Two studies met all 7 quality criteria and received 10 points, 3 studies got 9 points, 6 studies got 8 points, 16 studies received 7 points and 17 studies got 6 points. Thus, 44 of the 75 studies received 6 points or more and were evaluated to be of relatively high quality (18 longitudinal and 26 cross-sectional studies). The studies which received less than 6 points were mainly characterized by the following: not having a longitudinal design, the number of participants was below 100, a diagnostic evaluation of depressive disorders was not 
Table 1. Quality assessment

\begin{tabular}{|c|c|c|c|c|c|c|c|c|}
\hline Ref. No. & $\begin{array}{l}\text { Prospec- } \\
\text { tive } \\
\text { design }\end{array}$ & $\begin{array}{l}\text { Setting } \\
\text { defined }\end{array}$ & $\mathrm{n} \geq 100$ & $\begin{array}{l}\text { Diagnosis of } \\
\text { depression: } \\
\text { ICD/DSM }\end{array}$ & $\begin{array}{l}\text { Well-established } \\
\text { measure of } \\
\text { depression }\end{array}$ & $\begin{array}{l}\text { Well-established } \\
\text { measure of } \\
\text { coping }\end{array}$ & $\begin{array}{l}\text { Definition of coping } \\
\text { with reference to } \\
\text { literature }\end{array}$ & $\begin{array}{l}\text { Score +/- } \\
\max 10\end{array}$ \\
\hline \multicolumn{9}{|c|}{ Studies of 6 or more quality criteria } \\
\hline 26 & 1 & 1 & 1 & 2 & 2 & 2 & 1 & 10 \\
\hline 55 & 1 & 1 & 1 & 2 & 2 & 2 & 1 & 10 \\
\hline 66 & - & 1 & 1 & 2 & 2 & 2 & 1 & 9 \\
\hline 49 & - & 1 & 1 & 2 & 2 & 2 & 1 & 9 \\
\hline 56 & 1 & 1 & 1 & 2 & 2 & 2 & - & 9 \\
\hline 45 & 1 & 1 & 1 & - & 2 & 2 & 1 & 8 \\
\hline 50 & 1 & 1 & 1 & 2 & 2 & - & 1 & 8 \\
\hline 67 & 1 & 1 & - & 2 & 2 & 2 & - & 8 \\
\hline 68 & 1 & 1 & 1 & - & 2 & 2 & 1 & 8 \\
\hline 58 & - & 1 & - & 2 & 2 & 2 & 1 & 8 \\
\hline 59 & - & 1 & - & 2 & 2 & 2 & 1 & 8 \\
\hline 62 & - & 1 & 1 & - & 2 & 2 & 1 & 7 \\
\hline 47 & 1 & 1 & - & - & 2 & 2 & 1 & 7 \\
\hline 64 & - & 1 & 1 & - & 2 & 2 & 1 & 7 \\
\hline 69 & - & 1 & 1 & - & 2 & 2 & 1 & 7 \\
\hline 70 & 1 & 1 & 1 & - & 2 & 2 & - & 7 \\
\hline 71 & 1 & 1 & - & - & 2 & 2 & 1 & 7 \\
\hline 72 & 1 & 1 & 1 & - & 2 & 2 & - & 7 \\
\hline 66 & 1 & 1 & - & - & 2 & 2 & 1 & 7 \\
\hline 73 & 1 & 1 & 1 & - & 2 & 2 & - & 7 \\
\hline 52 & 1 & 1 & - & - & 2 & 2 & 1 & 7 \\
\hline 74 & - & 1 & 1 & - & 2 & 2 & 1 & 7 \\
\hline 75 & - & 1 & 1 & - & 2 & 2 & 1 & 7 \\
\hline 76 & - & 1 & 1 & - & 2 & 2 & 1 & 7 \\
\hline 77 & - & 1 & 1 & - & 2 & 2 & 1 & 7 \\
\hline 78 & - & 1 & 1 & - & 2 & 2 & 1 & 7 \\
\hline 79 & - & 1 & 1 & - & 2 & 2 & 1 & 7 \\
\hline 80 & 1 & 1 & 1 & - & 2 & - & 1 & 6 \\
\hline 25 & 1 & 1 & 1 & - & 2 & - & 1 & 6 \\
\hline 41 & - & 1 & 1 & 2 & 2 & - & - & 6 \\
\hline 81 & - & 1 & 1 & & 2 & 2 & - & 6 \\
\hline 35 & - & 1 & - & - & 2 & 2 & 1 & 6 \\
\hline 28 & - & 1 & - & - & 2 & 2 & 1 & 6 \\
\hline 60 & - & 1 & - & 2 & - & 2 & 1 & 6 \\
\hline 82 & - & 1 & 1 & - & 2 & 2 & - & 6 \\
\hline 83 & - & 1 & - & - & 2 & 2 & 1 & 6 \\
\hline 46 & - & 1 & 1 & - & 2 & 2 & - & 6 \\
\hline 84 & - & 1 & - & - & 2 & 2 & 1 & 6 \\
\hline 85 & 1 & 1 & 1 & - & 2 & - & 1 & 6 \\
\hline 86 & - & 1 & 1 & - & 2 & 2 & - & 6 \\
\hline 87 & - & 1 & 1 & - & 2 & 2 & - & 6 \\
\hline 88 & - & 1 & 1 & - & 2 & 2 & - & 6 \\
\hline 89 & - & 1 & 1 & - & 2 & 2 & - & 6 \\
\hline 57 & 1 & 1 & - & 2 & 2 & - & - & 6 \\
\hline
\end{tabular}


Table 1 (continued)

\begin{tabular}{|c|c|c|c|c|c|c|c|c|}
\hline Ref. No. & $\begin{array}{l}\text { Prospec- } \\
\text { tive } \\
\text { design }\end{array}$ & $\begin{array}{l}\text { Setting } \\
\text { defined }\end{array}$ & $n \geq 100$ & $\begin{array}{l}\text { Diagnosis of } \\
\text { depression: } \\
\text { ICD/DSM }\end{array}$ & $\begin{array}{l}\text { Well-established } \\
\text { measure of } \\
\text { depression }\end{array}$ & $\begin{array}{l}\text { Well-established } \\
\text { measure of } \\
\text { coping }\end{array}$ & $\begin{array}{l}\text { Definition of coping } \\
\text { with reference to } \\
\text { literature }\end{array}$ & $\begin{array}{l}\text { Score +/- } \\
\max 10\end{array}$ \\
\hline \multicolumn{9}{|c|}{ Studies of 5 or less quality criteria } \\
\hline 90 & - & 1 & 1 & - & - & 2 & 1 & 5 \\
\hline 61 & - & 1 & 1 & - & - & 2 & 1 & 5 \\
\hline 91 & 1 & 1 & 1 & - & - & 2 & - & 5 \\
\hline 92 & 1 & 1 & 1 & - & 2 & - & - & 5 \\
\hline 93 & 1 & 1 & - & - & - & 2 & 1 & 5 \\
\hline 94 & - & - & 1 & - & 2 & 2 & - & 5 \\
\hline 95 & - & 1 & 1 & - & 2 & - & 1 & 5 \\
\hline 96 & 1 & 1 & 1 & - & 2 & - & - & 5 \\
\hline 44 & - & 1 & - & - & 2 & 2 & - & 5 \\
\hline 53 & - & 1 & 1 & - & 2 & - & 1 & 5 \\
\hline 97 & - & 1 & - & - & 2 & 2 & - & 5 \\
\hline 98 & - & 1 & 1 & - & 2 & - & - & 4 \\
\hline 99 & - & 1 & - & - & 2 & - & 1 & 4 \\
\hline 36 & - & 1 & - & - & 2 & - & 1 & 4 \\
\hline 42 & - & 1 & - & - & 2 & - & 1 & 4 \\
\hline 100 & - & 1 & 1 & - & 2 & - & - & 4 \\
\hline 101 & - & - & - & - & 2 & 2 & - & 4 \\
\hline 43 & - & 1 & 1 & - & 2 & - & - & 4 \\
\hline 102 & - & 1 & 1 & - & 2 & - & - & 4 \\
\hline 40 & 1 & - & 1 & - & 2 & - & - & 4 \\
\hline 103 & - & 1 & 1 & - & - & - & 1 & 3 \\
\hline 104 & - & 1 & - & - & 2 & - & - & 3 \\
\hline 105 & - & 1 & - & - & 2 & - & - & 3 \\
\hline 48 & - & 1 & - & - & 2 & - & - & 3 \\
\hline 106 & - & 1 & 1 & - & - & - & 1 & 3 \\
\hline 107 & - & 1 & - & - & 2 & - & - & 3 \\
\hline 108 & - & 1 & - & - & - & 2 & - & 3 \\
\hline 109 & - & 1 & 1 & - & - & - & 1 & 3 \\
\hline 110 & - & 1 & - & - & 2 & - & - & 3 \\
\hline 111 & 1 & 1 & - & - & - & - & - & 2 \\
\hline 112 & - & 1 & - & - & - & - & - & 1 \\
\hline
\end{tabular}

applied, the use of well-established instruments to assess coping was not included and, lastly, a definition of the concepts of coping was not described with a reference to the literature (table 1 ).

\section{Assessment of Depression}

Information about depressive symptoms was obtained from self-report instruments, observation inventories, structural interviews, or from diagnostic evaluations applying the DSM-III/-R/IV criteria (table 4). A total of 21 different instruments were used in the studies to assess the symptoms of depression. The Center for Epidemiological Studies - Depression scale (CES-D) [31] was most often applied (24 studies). Four studies used a scale constructed for the specific study (table 4).

\section{Concepts and Assessments of Coping}

In all, 55 different measures of coping were applied in the 75 studies, and the instruments were found to be related theoretically with the following four clusters: (i) sense of coherence, (ii) various instruments of control orientation, (iii) coping strategies, style or actions (referred 
to as coping strategies hereafter), and (iv) religious coping. These clusters are again found to represent two different, but related parts of the coping process, resources and strategies of coping. The coping concepts, sense of coherence and control orientation, are both theoretically referred to as resources of coping [16, 32]. Religious coping may be understood both as a strategy of coping representing religious activities [24] and as resources of coping, when religiosity can serve as a system of values that may guide the individual's choices of coping strategies in times of stress $[33,34]$. The concept of religious coping is based on the theories of Folkman and Lazarus [19], and is thus related as strategies of coping [33].

In total, 9 studies included more than one of the four clusters of coping, and these were a combination of an instrument of control orientation and coping strategies in 7 studies and between control orientation and religious coping in 2 studies [35, 36] (tables 2, 3). The Sense of Coherence scale [37] was the most frequently used instrument and was applied in 9 studies (table 5). Among the total of 18 different instruments assessing control orientation, 3 instruments (the Internal/External Control of Reinforcement scale [15], the Multi-Health Locus of Control scale [38] and the Internal Locus of Control (mastery) [39]) were applied in 6 studies each (table 5). Five studies used instruments to assess control orientation specifically made for those studies. Regarding the 30 different instruments of coping strategies, the COPE inventory was applied most often, in 4 studies. Three studies used instruments of coping strategies specifically made for those studies. Among the 7 different instruments of religious coping, the Religious Coping index was most often applied, in 5 studies (table 5).

\section{Assessment of Coping in Samples of Older Persons}

No studies reported information about difficulties with administration of the coping instruments in older participants. However, 11 studies excluded participants with cognitive impairment, a diagnosis of dementia or major depression [40-50]. Three studies ( 1 from a nursing home, 1 community-based study and 1 study from a memory clinic) excluded items regarded as irrelevant to the situation of the participant [51-53]. Of the studies of older persons suffering from depression, cognitive impairment, or from nursing homes, 10 studies screened their participants for cognitive impairment, and 9 applied the Mini-Mental Status Examination (MMSE) [26, 35, 51, 54-61]. The criteria of exclusion varied from MMSE $\leq 6$ to 25 points, but most often a cutoff at 24 and 25 points was used.

\section{Older Persons Coping with Depression}

In the 44 studies with a quality score of 6 or more points, the main finding was of a strong association between resources and strategies of coping and depression, and this association appeared to be stable over time.

\section{Sense of Coherence}

All 9 studies using SOC were of higher quality (2 longitudinal and 7 cross-sectional studies; table 1). Of these 9 studies, 4 recruited participants from community settings and 5 recruited older persons from clinical settings of whom 3 studies were from somatic hospitals, including participants after suicide attempts ( 2 studies) and older persons with somatic disorders (1 study). Further, 1 study included depressed older persons from psychogeriatric clinics and 1 study included participants from nursing homes with different somatic disorders. These studies reported a negative relationship between SOC and depressive symptoms (tables 2,3). In the longitudinal studies, a higher total score on the SOC at baseline was related to fewer depressive symptoms at follow-up among participants, and was associated with a lower score on a depression scale in a nonremission group of depressed suicide attempters. The cross-sectional studies reported that higher total SOC scores were associated with lower scores on the depression scales. One study reported a negative association between SOC and 
Table 2. Longitudinal studies $(n=24)$

\begin{tabular}{|c|c|c|c|c|}
\hline $\begin{array}{l}\text { Ref. No. } \\
\text { (country) }\end{array}$ & Aim of study ${ }^{1}$ & Sample characteristics & $\begin{array}{l}\text { Instruments used to } \\
\text { assess depression }\end{array}$ & $\begin{array}{l}\text { Category }{ }^{2} \text { and instruments used } \\
\text { to assess coping }\end{array}$ \\
\hline \multicolumn{5}{|c|}{ Studies of 6 or more quality criteria } \\
\hline $\begin{array}{l}26 \\
\text { (USA) }\end{array}$ & $\begin{array}{l}\text { To study the relation between reli- } \\
\text { giousness and depression outcome } \\
\text { after considering clinical factors }\end{array}$ & $\begin{array}{l}114 \text { older patients from a psychogeriatric } \\
\text { clinical setting with major depression } \\
\text { MA }=67.4 \text { years, } S D=6.2 \text { years, } \\
63 \% \text { females }\end{array}$ & $\begin{array}{l}\text { DSM-IV } \\
\text { MADRS (T1-T2) }\end{array}$ & $\begin{array}{l}\text { Religious coping } \\
\text { Public religious activities (T1) } \\
\text { Private religious activities (T1) } \\
\text { RCOPE (T1) }\end{array}$ \\
\hline
\end{tabular}

\begin{tabular}{|c|c|c|c|c|}
\hline $\begin{array}{l}55 \\
\text { (USA) }\end{array}$ & $\begin{array}{l}\text { To examine the efficacy of augmenting } \\
\text { antidepressant medication and medi- } \\
\text { cation combined with dialectical } \\
\text { behavior therapy intervention for } \\
\text { adaptive coping }\end{array}$ & $\begin{array}{l}34 \text { older patients from a psychogeriatric } \\
\text { clinical setting with depression } \\
\text { MA }=66 \text { years, } S D=5 \text { years, } 85 \% \text { females }\end{array}$ & $\begin{array}{l}\text { Ham-D (T1-T3) } \\
\text { DDES (T1-T3) } \\
\text { BDI (T1-T3) } \\
\text { DSM-III, DSM-III-R, } \\
\text { DSM-IV }\end{array}$ & $\begin{array}{l}\text { Coping strategies } \\
\text { CSQ (T1-T3) }\end{array}$ \\
\hline $\begin{array}{l}56 \\
\text { (Sweden) }\end{array}$ & $\begin{array}{l}\text { To investigate } 1 \text {-year outcomes in } \\
\text { suicide attempters aged } 70+\text { and to } \\
\text { identify predictors of these outcomes }\end{array}$ & $\begin{array}{l}101 \text { older patients from somatic health } \\
\text { care setting hospitalized after a suicide } \\
\text { attempt } \\
\text { MA }=79.7 \text { years, } S D=4,9,31,7 \% \\
\text { females }\end{array}$ & $\begin{array}{l}\text { DSM-IV (T1+T2) } \\
\text { MADRS (T1+T2) }\end{array}$ & $\begin{array}{l}\text { Sense of coherence } \\
\text { SOC-29 (T1+T2) }\end{array}$ \\
\hline $\begin{array}{l}45 \\
\text { (Germany) }\end{array}$ & $\begin{array}{l}\text { To determine the relation between } \\
\text { self-perceived health, SOC and mea- } \\
\text { sures of subjective well-being and } \\
\text { depression }\end{array}$ & $\begin{array}{l}74 \text { older patients from a somatic health } \\
\text { care setting, hospitalized for geriatric } \\
\text { disorders } \\
\text { MA }=77.4 \text { years, } S D=6,7,63,5 \% \\
\text { females }\end{array}$ & HADS (T1+T2) & $\begin{array}{l}\text { Sense of coherence } \\
\text { SOC-29 (T1+T2) }\end{array}$ \\
\hline $\begin{array}{l}50 \\
\text { (USA) }\end{array}$ & $\begin{array}{l}\text { To identify the predictors of late-life } \\
\text { depression that are amenable to } \\
\text { change and may lead to interventions } \\
\text { resulting in better and faster remis- } \\
\text { sion }\end{array}$ & $\begin{array}{l}271 \text { older patients from a somatic health } \\
\text { care setting, treated for depression } \\
<59 \text { years }\end{array}$ & $\begin{array}{l}\text { DSM-IV } \\
(\mathrm{T} 1+\mathrm{T} 2+\mathrm{T} 3+\mathrm{T} 4+\mathrm{T} 5+\mathrm{T} 6) \\
\text { HRSD } \\
\text { (T1+T2+T3+T4+T5+T6) }\end{array}$ & $\begin{array}{l}\text { Control orientation } \\
\text { The Optimization in Primary } \\
\text { and Secondary Control survey } \\
(\mathrm{T} 1+\mathrm{T} 2+\mathrm{T} 3+\mathrm{T} 4+\mathrm{T} 5+\mathrm{T} 6)\end{array}$ \\
\hline $\begin{array}{l}67 \\
\text { (Aus- } \\
\text { tralia) }\end{array}$ & $\begin{array}{l}\text { To study exercise as an antidepres- } \\
\text { sant and to explore relations between } \\
\text { participant characteristics and treat- } \\
\text { ment result }\end{array}$ & $\begin{array}{l}60 \text { older patients from a somatic health } \\
\text { care setting included in a randomized } \\
\text { controlled trial with depression } \\
\geq 60 \text { years, } 55 \% \text { females } \\
\text { High-intensity training group }(n=20) \\
\text { Low-intensity training group }(n=20) \\
\text { Receiving GP care }(n=20)\end{array}$ & $\begin{array}{l}\text { DSM-IV } \\
\text { Ham-D (T1-T2) } \\
\text { GDS (T1-T2) }\end{array}$ & $\begin{array}{l}\text { Control orientation } \\
\text { Outcome expectation (T1) } \\
\text { MHLC (T1) } \\
\text { Self-Efficacy scale (T1) }\end{array}$ \\
\hline $\begin{array}{l}113 \\
\text { (USA) }\end{array}$ & $\begin{array}{l}\text { To examine whether LOC beliefs } \\
\text { buffer or mediate the impact of } \\
\text { chronic financial strain on depression }\end{array}$ & $\begin{array}{l}351 \text { older community residents } \\
M A=73.4 \text { years, } S D=6.2 \text { years, } 66 \% \\
\text { females }\end{array}$ & CES-D (T1-T2) & $\begin{array}{l}\text { Control orientation } \\
\text { I/E scale (T1-T2) }\end{array}$ \\
\hline $\begin{array}{l}47 \\
(\mathrm{UK})\end{array}$ & $\begin{array}{l}\text { To study if perceived control belief } \\
\text { was associated with depression and if } \\
\text { depression mediated this effect }\end{array}$ & $\begin{array}{l}71 \text { older patients from a somatic health } \\
\text { care setting within } 3 \text { weeks of an acute } \\
\text { stroke } \\
\text { MA }=69.4 \text { years, SD = } 9.2 \text { years, } \\
49.3 \% \text { females }\end{array}$ & HAD (T1-T3) & $\begin{array}{l}\text { Control orientation } \\
\text { Coping strategies } \\
\text { RLOC (T1-T3) } \\
\text { Exercise coping (T1-T3) }\end{array}$ \\
\hline
\end{tabular}




\begin{tabular}{ll} 
Time of assessment & Findings at baseline \\
& \\
T1: Baseline & $\begin{array}{l}\text { Higher levels of public religious } \\
\text { practice and positive religious } \\
\text { coping were associated with } \\
\text { reduced depressive score, and } \\
\text { low negative religious coping } \\
\text { was associated with decreased } \\
\text { depressive score }\end{array}$ \\
\hline
\end{tabular}

T1: pretreatment
T2: 28 weeks
posttreatment
T3: 6 months
T1 = 11 median days
after suicide attempt
$\mathrm{T} 2=391$ median

days after baseline
Findings at follow-up

Higher levels of positive religious coping at $\mathrm{T} 1$ were associated with lower depressive scores at $\mathrm{T} 2$
Findings of no relation
Comments on method

\begin{tabular}{ll}
\hline $\mathrm{T} 1=$ Baseline & A higher total SoC score at \\
$\mathrm{T} 2=5$ years & $\mathrm{T} 1$ was correlated with \\
& reduced depressive symp- \\
& toms at $\mathrm{T} 2$
\end{tabular}

Patients receiving medication + dialectical behavioral therapy showed higher adaptive coping from $\mathrm{T} 1$ to T2 Higher total SOC score and lower depression at $\mathrm{T} 1$ were associated factors in non-remission group at T2
Higher levels of public religious practice, private religious practice and negative religious coping at $\mathrm{T} 1$ were not related to lower depression score at T2

Multivariate regression analysis Adjusted for age, gender, education, social support, number of depressed episodes and ECT both at baseline and follow-up

There was no relation between group difference at T2 or T3 in adaptive coping
Repeated-measures ANOVAs Modified Bonferroni correction 2-tailed, paired t test. Fisher's exact test. Multivariate logistic regression analyses.

\begin{tabular}{|c|c|c|c|c|}
\hline $\begin{array}{l}\mathrm{T} 1=\text { baseline } \\
\mathrm{T} 2=4 \text { months } \\
\mathrm{T} 3=8 \text { months } \\
\mathrm{T} 4=12 \text { months } \\
\mathrm{T} 5=16 \text { months } \\
\mathrm{T} 6=24 \text { months }\end{array}$ & $\begin{array}{l}\text { Persons with high anxiety and } \\
\text { engagement had lower depres- } \\
\text { sion severity than those with } \\
\text { high anxiety and low engage- } \\
\text { ment }\end{array}$ & $\begin{array}{l}\text { Greater disengagement } \\
\text { (from unattainable health } \\
\text { goals) at baseline predicted } \\
\text { shorter time before remis- } \\
\text { sion }\end{array}$ & $\begin{array}{l}\text { Use of engagement strate- } \\
\text { gies was not associated } \\
\text { with time before depres- } \\
\text { sion remission }\end{array}$ & $\begin{array}{l}\text { Pearson's partial correlation } \\
\text { Adjusted for depression severity. } \\
\text { Univariate linear regression. } \\
\text { Multivariate cox proportional } \\
\text { hazard models (adjusting for age, } \\
\text { gender, intervention, physical } \\
\text { illness, baseline depression score) }\end{array}$ \\
\hline $\begin{array}{l}\text { T2: } 8 \text { weeks after } \\
\text { intervention }\end{array}$ & & $\begin{array}{l}\text { A high outcome expectation } \\
\text { at T1 was related to reduc- } \\
\text { tion in depressive score in } \\
\text { the low intensity training } \\
\text { group at T2 }\end{array}$ & $\begin{array}{l}\text { LOC and self-efficacy at } \\
\text { baseline were not related } \\
\text { to reduction of depressive } \\
\text { symptoms at follow-up } \\
\text { A high baseline outcome } \\
\text { expectation was not } \\
\text { related with reduction in } \\
\text { depressive score in the } \\
\text { other groups at follow-up }\end{array}$ & $\begin{array}{l}\text { Comparison between the groups } \\
\text { (ANCOVA and ANOVA) }\end{array}$ \\
\hline $\begin{array}{l}\text { T1: Baseline } \\
\text { T2: } 1 \frac{1 / 2}{2} \text { years } \\
\text { (mean } 18.7 \\
\text { SD }=1.9 \text { months) } \\
(\mathrm{n}=265)\end{array}$ & & $\begin{array}{l}\text { LOC at T1 buffers the effect } \\
\text { of chronic financial strain } \\
\text { on depression } \\
\text { Stronger internal control } \\
\text { belief at T2 is associated } \\
\text { with reduced impact of } \\
\text { financial strain at } \\
\text { depressed affect at T2 }\end{array}$ & & $\begin{array}{l}\text { Hierarchical multiple linear } \\
\text { regression analysis } \\
\text { Adjusted for fanancial strain }\end{array}$ \\
\hline $\begin{array}{l}\text { T1: } 10-12 \text { days after } \\
\text { stroke } \\
\text { T2: } 1 \text { month after } \\
\text { discharge } \\
\text { T3: } 6 \text { months after } \\
\text { discharge }\end{array}$ & & $\begin{array}{l}\text { Higher perceived control } \\
\text { (at T2 and T3) correlated } \\
\text { moderately with reduced } \\
\text { depressive score at } \mathrm{T} 2 \text { and } \\
\mathrm{T} 3 \text {, but was not mediated } \\
\text { by depression }\end{array}$ & & $\begin{array}{l}\text { Correlations } \\
\text { Regression analysis }\end{array}$ \\
\hline
\end{tabular}


Table 2 (continued)

\begin{tabular}{|c|c|c|c|c|}
\hline $\begin{array}{l}\text { Ref. No. } \\
\text { (country) }\end{array}$ & Aim of study ${ }^{1}$ & Sample characteristics & $\begin{array}{l}\text { Instruments used to } \\
\text { assess depression }\end{array}$ & $\begin{array}{l}\text { Category }{ }^{2} \text { and instruments used } \\
\text { to assess coping }\end{array}$ \\
\hline $\begin{array}{l}70 \\
\text { (USA) }\end{array}$ & $\begin{array}{l}\text { To describe the natural history of } \\
\text { adaptation to stroke and to identify } \\
\text { survivor predictors of depressive } \\
\text { symptoms }\end{array}$ & $\begin{array}{l}53 \text { older patients from a somatic health } \\
\text { care setting receiving acute rehabilita- } \\
\text { tion after stroke } \\
\text { MA = } 60.1 \text { years, SD = } 11.9 \text { years, } \\
37 \% \text { females }\end{array}$ & CES-D (T1-T4) & $\begin{array}{l}\text { Coping strategies, } \\
\text { control orientation } \\
\text { Cognitive appraisal (T1-T4) } \\
\text { WOCQ (T1-T4) } \\
\text { LOT }(\mathrm{T} 1-\mathrm{T} 2, \mathrm{~T} 4)\end{array}$ \\
\hline
\end{tabular}

\begin{tabular}{|c|c|c|c|c|}
\hline $\begin{array}{l}71 \\
\text { (Canada) }\end{array}$ & $\begin{array}{l}\text { To describe changes in the adaption } \\
\text { process for stroke survivors and } \\
\text { spouses for appraisal and coping, and } \\
\text { identifying domains of the adaption } \\
\text { process related to depression }\end{array}$ & $\begin{array}{l}88 \text { older patients from a somatic health } \\
\text { care setting, assessed within the first } 2 \\
\text { weeks poststroke } \\
\text { MA }=71.8 \text { years, } S D=10.8 \text { years, } 37 \% \\
\text { females } \\
47 \text { spouses } \\
\text { MA }=69.2 \text { years, } S D=11.2,76.6 \% \\
\text { females }\end{array}$ & BDI (T3) & $\begin{array}{l}\text { Coping strategies } \\
\text { SAM (T1-T3) } \\
\text { RWCQ (T1-T3) }\end{array}$ \\
\hline
\end{tabular}

\begin{tabular}{llll}
\hline 72 & To study the impact of known predic- & 110 older community residents with & CES-D (T1-T2) \\
(USA) & tor variables (among coping) for & depressive symptoms & Control orientation \\
& depression within an urban popula- & $\geq 55$ years, $52 \%$ females & Mastery scale (T1)
\end{tabular}
tion

\begin{tabular}{ll}
\hline 114 & To examine the relation between \\
(Nether- & $\begin{array}{l}\text { cognitive coping strategies and } \\
\text { depressive symptoms in old age when }\end{array}$ \\
lands) & $\begin{array}{l}\text { adjusting for depressive symptoms } \\
\text { over time }\end{array}$
\end{tabular}

\author{
99 older community residents \\ $\mathrm{MA}=77.2$ years, $\mathrm{SD}=6.1$ years, $48 \%$ \\ females
}

GDS (T1-T2) over time living accommodation

MA = 86.7 years, $64 \%$ females

\begin{tabular}{|c|c|c|c|c|}
\hline $\begin{array}{l}73 \\
\text { (Sweden) }\end{array}$ & $\begin{array}{l}\text { To examine change in depressive } \\
\text { symptoms over time and study corre- } \\
\text { lates of depressive symptoms by } \\
\text { known risk factors and mastery }\end{array}$ & $\begin{array}{l}254 \text { of the oldest old community resi- } \\
\text { dents in municipalities, independent of } \\
\text { living accommodation } \\
\text { MA = } 86.7 \text { years, } 64 \% \text { females }\end{array}$ & CES-D (T1-T2) & $\begin{array}{l}\text { Control orientation } \\
\text { Mastery scale (T1) }\end{array}$ \\
\hline $\begin{array}{l}52 \\
\text { (USA) }\end{array}$ & $\begin{array}{l}\text { To study what group of older adults } \\
\text { benefits most from a control-enhanc- } \\
\text { ing intervention and whether LOC } \\
\text { moderates the benefit derived }\end{array}$ & $\begin{array}{l}27 \text { older patients from a nursing home } \\
\text { setting randomized for having the oppor- } \\
\text { tunity to care for a plant }(10 \text { older per- } \\
\text { sons) or standard care }(17 \text { older per- } \\
\text { sons) } \\
\text { MA = } 81 \text { years, SD = } 9.29 \text { years, } 70 \% \\
\text { females }\end{array}$ & GDS SF (T1-T2) & $\begin{array}{l}\text { Control orientation } \\
\text { I/E scale (T1) }\end{array}$ \\
\hline $\begin{array}{l}80 \\
\text { (Australia) }\end{array}$ & $\begin{array}{l}\text { To study if SOC mediated the relation } \\
\text { between driving status and depres- } \\
\text { sive symptoms }\end{array}$ & $\begin{array}{l}700 \text { older community residents } \\
\mathrm{MA}=75.1 \text { years, } \mathrm{SD}=4.7 \text { years, } 27.4 \% \\
\text { females }\end{array}$ & $\begin{array}{l}\text { CES-D } \\
\text { (T1-T2) }\end{array}$ & $\begin{array}{l}\text { Control orientation } \\
\text { Desired Control Measure (T1- } \\
\text { T2) }\end{array}$ \\
\hline
\end{tabular}

Coping strategies

Cognitive Emotion Regulation

questionnaire (T2) 


\begin{tabular}{|c|c|c|c|c|}
\hline Time of assessment & Findings at baseline & Findings at follow-up & Findings of no relation & Comments on method \\
\hline $\begin{array}{l}\text { T1: prior to discharge } \\
\text { from acute rehabilita- } \\
\text { tion } \\
\text { T2: } 6-10 \text { weeks post- } \\
\text { discharge to home } \\
\text { T3: } 12 \text { months post- } \\
\text { discharge } \\
\text { T4: } 2 \text { years postdis- } \\
\text { charge }\end{array}$ & $\begin{array}{l}\text { Higher levels of finding meaning } \\
\text { and lower avoidance coping } \\
\text { were associated with lower } \\
\text { levels of depressive symptoms }\end{array}$ & $\begin{array}{l}\text { Higher score of cognitive } \\
\text { appraisal of support at T1 } \\
\text { was associated with lower } \\
\text { depressive symptoms at T4 }\end{array}$ & $\begin{array}{l}\text { Optimism (Life Orienta- } \\
\text { tion test) at T1 was not } \\
\text { associated with depres- } \\
\text { sive symptoms at T4 }\end{array}$ & $\begin{array}{l}\text { Hierarchical regression analysis } \\
\text { Adjusted for lower levels of sup- } \\
\text { port at T1 } \\
\text { Hierarchical analysis adjusted for } \\
\text { degree of illness severity and } \\
\text { depressive score at T1 } \\
\text { Hierarchical analysis. Adjusted for } \\
\text { illness severity, social environ- } \\
\text { mental situation and cognitive } \\
\text { appraisal of impact at T4, Life } \\
\text { Orientation test (excluded from } \\
\text { analysis) }\end{array}$ \\
\hline $\begin{array}{l}\text { T1: } 2 \text { weeks post- } \\
\text { stroke } \\
\text { T2: } 3 \text { months } \\
\text { T3: } 6 \text { months } \\
\text { poststroke }\end{array}$ & & $\begin{array}{l}\text { Higher challenge and lower } \\
\text { centrality scores of the } \\
\text { primary appraisal measure } \\
\text { at T1 for patients and } \\
\text { spouses were associated } \\
\text { with lower depressive } \\
\text { score at T3 }\end{array}$ & $\begin{array}{l}\text { Secondary appraisal, } \\
\text { experience of stressful- } \\
\text { ness and the other coping } \\
\text { variables at T1 were not } \\
\text { associated with depres- } \\
\text { sive symptoms at T3 }\end{array}$ & $\begin{array}{l}\text { General linear model of repeated } \\
\text { measures } \\
\text { Pearson's correlation } \\
\text { Multiple linear regression model }\end{array}$ \\
\hline $\begin{array}{l}\text { T1: Baseline } \\
\text { T2: } 1-4.5 \text { years } \\
\text { Mean } 3.0 \text { years }\end{array}$ & & $\begin{array}{l}\text { A lower sense of helpless- } \\
\text { ness (higher internal LOC) } \\
\text { at T1 was associated with } \\
\text { lower risk for depression at } \\
\text { T2 }\end{array}$ & & $\begin{array}{l}\text { Multiple logistic regression analy- } \\
\text { sis } \\
\text { Adjusted for age, gender, race, } \\
\text { household income, depressive } \\
\text { score at baseline, IADL, Physical } \\
\text { illness, use of mental health } \\
\text { resources during follow-up }\end{array}$ \\
\hline $\begin{array}{l}\text { T1: Baseline } \\
\text { T2: } 2 \text { years }\end{array}$ & $\begin{array}{l}\text { The cognitive coping strategy } \\
\text { high acceptance and positive } \\
\text { reappraisal at T2 were associ- } \\
\text { ated with less depressive symp- } \\
\text { toms at T2 }\end{array}$ & & $\begin{array}{l}\text { Self-blame, positive refo- } \\
\text { cusing, refocus on plan- } \\
\text { ning, putting into perspec- } \\
\text { tive or other-blame were } \\
\text { not correlated with } \\
\text { depression scores } \\
\text { In adjusted analysis, } \\
\text { rumination and being } \\
\text { catastrophic were not } \\
\text { associated with depres- } \\
\text { sive symptoms }\end{array}$ & $\begin{array}{l}\text { Pearson's correlation and Multi- } \\
\text { variate regression analysis } \\
\text { Adjusted for depressive symptoms } \\
\text { at baseline, earlier and recent life } \\
\text { events, and the coping strategies } \\
\text { rumination and being catastrophic } \\
\text { at follow-up }\end{array}$ \\
\hline $\begin{array}{l}\text { T1: } \text { Baseline } \\
\text { T2: } 2 \text { years }(n=213)\end{array}$ & $\begin{array}{l}\text { High feeling of mastery at T1 } \\
\text { was associated with lower levels } \\
\text { of depressive symptoms and } \\
\text { mediated the importance of low } \\
\text { cognition and ADL for high level } \\
\text { of depressive symptoms }\end{array}$ & $\begin{array}{l}\text { Elderly with depression at } \\
\text { 2-year follow-up had lower } \\
\text { levels of mastery at T1, } \\
\text { compared with elderly } \\
\text { without depression }\end{array}$ & & $\begin{array}{l}\text { Path analysis } \\
\text { One-way ANOVA }\end{array}$ \\
\hline $\begin{array}{l}\text { T1: few weeks before } \\
\text { intervention } \\
\text { T2: } 2 \text { weeks after } \\
\text { intervention }\end{array}$ & & & $\begin{array}{l}\text { LOC did not moderate the } \\
\text { effect of control-enhanc- } \\
\text { ing intervention on } \\
\text { depression }\end{array}$ & Three-way ANOVA \\
\hline $\begin{array}{l}\text { T1: Baseline } \\
\text { T2: } 2 \text { years }\end{array}$ & $\begin{array}{l}\text { A low externally oriented expec- } \\
\text { tancy of control was associated } \\
\text { with lower depressive score }\end{array}$ & $\begin{array}{l}\text { Drivers had both lower } \\
\text { depressive score and exter- } \\
\text { nal expectancy of control, } \\
\text { than ceased drivers both at } \\
\text { T1 and at T2 } \\
\text { A high external perceived } \\
\text { control mediated the asso- } \\
\text { ciation between driving } \\
\text { status and depressive } \\
\text { symptoms }\end{array}$ & & $\begin{array}{l}\text { Multiple regression analysis } \\
\text { 2-group comparison } \\
\text { Multilevel general linear models } \\
\text { Adjusted for age, gender, educa- } \\
\text { tion, marital status, alone, income } \\
\text { and health }\end{array}$ \\
\hline
\end{tabular}


Table 2 (continued)

\begin{tabular}{|c|c|c|c|c|}
\hline $\begin{array}{l}\text { Ref. No. } \\
\text { (country) }\end{array}$ & Aim of study ${ }^{1}$ & Sample characteristics & $\begin{array}{l}\text { Instruments used to } \\
\text { assess depression }\end{array}$ & $\begin{array}{l}\text { Category }{ }^{2} \text { and instruments used } \\
\text { to assess coping }\end{array}$ \\
\hline $\begin{array}{l}25 \\
\text { (USA) }\end{array}$ & $\begin{array}{l}\text { To study the frequency of religious } \\
\text { coping and the relation between this } \\
\text { behavior and depression }\end{array}$ & $\begin{array}{l}850 \text { older patients from a somatic health } \\
\text { care setting admitted to medical or } \\
\text { neurological services at a medical center } \\
M A=69.8 \text { years, } S D=4.9 \text { years, } 0 \% \\
\text { females }\end{array}$ & $\begin{array}{l}\text { GDS (T1-T2) } \\
\text { Ham-D (T1) }\end{array}$ & $\begin{array}{l}\text { Religious coping } \\
\text { Religious coping (T1-T2) }\end{array}$ \\
\hline
\end{tabular}

\begin{tabular}{|c|c|c|c|c|}
\hline $\begin{array}{l}85 \\
\text { (Germany) }\end{array}$ & $\begin{array}{l}\text { To evaluate the predictive power and } \\
\text { moderating effects of coping, life } \\
\text { orientation and control on depression }\end{array}$ & $\begin{array}{l}272 \text { older patients from a somatic health } \\
\text { care setting, with bypass operation } \\
\text { MA = } 61.2 \text { years, } S D=8.6 \text { years, } 15 \% \\
\text { females }\end{array}$ & POMS (T1-T2) & $\begin{array}{l}\text { Coping strategies, } \\
\text { control orientation } \\
\text { CCQ (T1-T2) } \\
\text { HLC (T1) } \\
\text { Generalized self-efficacy beliefs } \\
\text { (T1-T2) } \\
\text { LOT (T1-T2) }\end{array}$ \\
\hline $\begin{array}{l}57 \\
\text { (USA) }\end{array}$ & $\begin{array}{l}\text { To examine the relation between } \\
\text { suppression of unpleasant thoughts } \\
\text { and the outcome of treatment }\end{array}$ & $\begin{array}{l}34 \text { older patients from a psychogeriatric } \\
\text { setting with depression } \\
\text { MA = } 66 \text { years, } 0 \% \text { females }\end{array}$ & $\begin{array}{l}\text { DSM-III-R } \\
\text { BDI (T1-T3) }\end{array}$ & $\begin{array}{l}\text { Coping strategies } \\
\text { WBSI (T1-T3) }\end{array}$ \\
\hline
\end{tabular}

Studies of 6 or less quality criteria

396 older patients from a somatic health RDC (T1-T2) care setting, with cardiac illness, 325 with chronic illness

$\mathrm{MA}=61.8$ years, $\mathrm{SD}=3.2$ years, $23.7 \%$

females

75 with acute illness

$\mathrm{MA}=61.1$ years, $\mathrm{SD}=2.9$ years, $26.8 \%$

females

\begin{tabular}{ll}
\hline 92 & $\begin{array}{l}\text { To study factors associated with } \\
\text { degree of depression }\end{array}$ \\
& \\
\hline 93 & $\begin{array}{l}\text { To study the effect of giving older } \\
\text { persons in an institution partial con- } \\
\text { trol over the activities program }\end{array}$
\end{tabular}

\begin{tabular}{ll}
\hline 96 & To evaluate whether osteoarthritis \\
(Canada) & pain determines depressed mood
\end{tabular}

123 older patients from a somatic health care setting, with poststroke depression $\mathrm{MA}=66$ years, $\mathrm{SD}=13.6$ years, $48.8 \%$ females

64 older patients from a somatic health care setting, in a medical convalescent hospital MA $=73.1$ years knee osteoarthritis
Coping strategies CRI (T2)

\section{4 older community residents with hip/ CES-D (T1+T2+T3) Coping strategies}

$\mathrm{MA}=75.4$ years, range 56.7-96 years, Behavioral Coping scale (T1) $78,5 \%$ females

Pain Catastrophizing scale (T1) $\begin{array}{ll}\text { BDI (T1-T3) } & \text { Control orientation } \\ \text { WDI (T1-T3) } & \text { RLOC (T2-T3) }\end{array}$

Rated by staff members Control orientation (T1-T2) $\quad$ LOC (T1-T2) Control orientation HLC (T1)
1,164 older patients from a somatic health care setting, recruited from 2 GPs

$\geq 65$ years

GDS (T1-T2) these in relation to persistent depression

\begin{tabular}{|c|c|c|c|c|}
\hline $\begin{array}{l}111 \\
\text { (Sweden) }\end{array}$ & $\begin{array}{l}\text { To assess the role of LOC in improving } \\
\text { mood }\end{array}$ & $\begin{array}{l}30 \text { older community residents from a } \\
\text { senior citizen apartment building } \\
\text { MA }=77.6 \text { years, } 66.7 \% \text { females }\end{array}$ & Mood scale (T1+T3) & $\begin{array}{l}\text { Control orientation } \\
\text { LOC (T1) }\end{array}$ \\
\hline
\end{tabular}




\begin{tabular}{|c|c|c|c|c|}
\hline Time of assessment & Findings at baseline & Findings at follow-up & Findings of no relation & Comments on method \\
\hline $\begin{array}{l}\text { T1: Baseline } \\
\text { T2: } \\
\text { within } 21 \text { months } \\
\text { (mean } 6 \text { months) } \\
\text { (n = 256) }\end{array}$ & $\begin{array}{l}\text { Higher score of religious coping } \\
\text { was associated with lower } \\
\text { depressive score }\end{array}$ & $\begin{array}{l}\text { Higher score of religious } \\
\text { coping at } \mathrm{T} 1 \text { was associated } \\
\text { with lower depressive } \\
\text { score at T2 }\end{array}$ & $\begin{array}{l}\text { Religious coping index } \\
\text { score at T1 was not asso- } \\
\text { ciated with depression } \\
\text { score at T2 } \\
\text { No relation was found } \\
\text { between change in reli- } \\
\text { gious coping index and } \\
\text { depressive symptoms }\end{array}$ & $\begin{array}{l}\text { Linear regression analysis } \\
\text { Adjusted for functional status and } \\
\text { religious affiliation } \\
\text { Pearson's correlation } \\
\text { Linear regression analysis } \\
\text { Adjusted for depression at } \mathrm{T} 1 \text { and } \\
\text { medical diagnosis }\end{array}$ \\
\hline $\begin{array}{l}\text { T1: } 1 \text { week after } \\
\text { surgery } \\
\text { T2: } 6 \text { months }\end{array}$ & $\begin{array}{l}\text { Higher coping competence and } \\
\text { higher score on Life orientation } \\
\text { test were independently associ- } \\
\text { ated with lower depressive } \\
\text { score }\end{array}$ & $\begin{array}{l}\text { Higher belief in powerful } \\
\text { others at T1 was associated } \\
\text { with lower depressive } \\
\text { score at T2 } \\
\text { High coping competence } \\
\text { had a moderating effect on } \\
\text { the association between } \\
\text { high depressive score and a } \\
\text { high level of stress }\end{array}$ & & $\begin{array}{l}\text { Hierarchical regression analysis } \\
\text { Adjusted for health LOC variables }\end{array}$ \\
\hline $\begin{array}{l}\text { T1: pretreatment } \\
\text { T2: } 28 \text { weeks post- } \\
\text { treatment } \\
\text { T3: } 6 \text { months post- } \\
\text { treatment }\end{array}$ & & $\begin{array}{l}\text { Patients with lower thought } \\
\text { suppression of unpleasant } \\
\text { thoughts at T1 had lower } \\
\text { depressive symptoms than } \\
\text { patients with high suppres- } \\
\text { sion at T3 }\end{array}$ & $\begin{array}{l}\text { Level of thought suppres- } \\
\text { sion at T1 was not associ- } \\
\text { ated with the depressive } \\
\text { score at T2 }\end{array}$ & $\begin{array}{l}\text { Multivariate tests } \\
\text { Adjusted for depression score at } \\
\text { baseline and age at onset of } \\
\text { depression }\end{array}$ \\
\hline $\begin{array}{l}\text { T1: Baseline } \\
\text { T2: } 1 \text { year }\end{array}$ & & $\begin{array}{l}\text { More approach coping (ver- } \\
\text { sus avoidance coping) at T1 } \\
\text { was significantly associated } \\
\text { with lower depressive } \\
\text { symptom score at T2 }\end{array}$ & & $\begin{array}{l}\text { LISREL test } \\
\text { Adjusted for social support and } \\
\text { depression score at T1 }\end{array}$ \\
\hline $\begin{array}{l}\text { T1: } 1 \text { month poststroke } \\
\text { T2: } 3 \text { months } \\
\text { T3: } 6 \text { months }(n=112)\end{array}$ & & $\begin{array}{l}\text { Higher Recovery LOC score } \\
\text { at T2 was associated with } \\
\text { reduced risk for depression } \\
\text { at T3 }\end{array}$ & & $\begin{array}{l}\text { Multiple logistic regression analy- } \\
\text { ses } \\
\text { Adjusted for acquired language } \\
\text { disorder }\end{array}$ \\
\hline $\begin{array}{l}\text { T1: Before treatment } \\
\text { T2: } 60 \text { days after } \\
\text { intervention }\end{array}$ & & $\begin{array}{l}\text { The LOC scores indicated } \\
\text { higher internal LOC after } \\
\text { intervention }\end{array}$ & $\begin{array}{l}\text { The depression score } \\
\text { was not changed after } \\
\text { intervention }\end{array}$ & $\begin{array}{l}\text { Paired t test between pre- and } \\
\text { post-test }\end{array}$ \\
\hline $\begin{array}{l}\mathrm{T} 1=\text { baseline } \\
\mathrm{T} 2=12 \text { months } \\
\mathrm{T} 3=24 \text { months }\end{array}$ & $\begin{array}{l}\text { Higher use of coping behaviors } \\
\text { was associated with lower } \\
\text { depressive symptoms }\end{array}$ & & $\begin{array}{l}\text { Pain catastrophizing was } \\
\text { not associated with } \\
\text { depressed mood }\end{array}$ & Correlation and path analysis \\
\hline $\begin{array}{l}\text { T1: Baseline } \\
\text { T2: } 2 \text { years }\end{array}$ & & $\begin{array}{l}\text { Having medium high or } \\
\text { high belief in powerful } \\
\text { others at } \mathrm{T} 1 \text { reduced the } \\
\text { odds for persistence of } \\
\text { depression at T2 }\end{array}$ & & $\begin{array}{l}\text { Logistic regression analyses } \\
\text { Adjusted for age, gender, baseline } \\
\text { depression, and general health, } \\
\text { dissatisfaction with support and } \\
\text { loneliness at follow-up }\end{array}$ \\
\hline $\begin{array}{l}\text { T1: Before treatment } \\
\text { T2: } 3 \text { months } \\
\text { T3: } 6 \text { months } \\
\text { after social activation } \\
\text { intervention }\end{array}$ & & $\begin{array}{l}\text { Lower level of internal } \\
\text { control at T1 was associ- } \\
\text { ated with improvement of } \\
\text { mood at T3 }\end{array}$ & & $\begin{array}{l}\text { Multiple linear regression analysis } \\
\text { Adjusting for marital status, social } \\
\text { activity, neurotic personality traits }\end{array}$ \\
\hline
\end{tabular}

${ }^{1}$ Aim of the published studies was in relation to the purpose of this review study. ${ }^{2}$ The 4 main categories of coping: coping strategies (also including style and actions), control orientation, sense of coherence and religious coping. 
Table 3. Cross-sectional studies $(n=51)$

\begin{tabular}{lll}
\hline $\begin{array}{l}\text { Ref. No. Aim of the study } \\
\text { (country) }\end{array}$ & $\begin{array}{l}\text { Sample characteristics } \\
\text { instruments used } \\
\text { to assess depres- } \\
\text { sion }\end{array}$
\end{tabular}

Studies of 6 and more quality criteria

$65 \quad$ To examine the joint effects of stress and coping

(Netherlands) resources in predicting depressive symptoms
194 older community residents randomly selected from

GDS metropolitan area

$\mathrm{MA}=76.5$ years, $\mathrm{SD}=7.25$ years, $52 \%$ females

80 older patients from a somatic health care setting with

DSM-IV

\section{9}

To examine the relation between coping styles and depressive symptoms heart failure and depressive symptoms

GDS

MA $=69$ years, $52 \%$ females

86 older patients from a psychogeriatric clinical setting with

major depression

$\mathrm{MA}=76.6$ years, $\mathrm{SD}=7.4$ years, $70.9 \%$ females
58 To determine the relation between religious practices and physical, social, and cognitive variables known to influence the prognosis of depression in the elderly

DSM-IV

31 older patients from a psychogeriatric clinical setting

DSM-IV

59 To examine the association between cognitive problem (Israel) solving and SOC with IADL function and depression

with depression

GDS-15
$\mathrm{MA}=73$ years, $\mathrm{SD}=9.3$

30 older community residents without depression

$\mathrm{MA}=78$ years, $\mathrm{SD}=5.8$

\begin{tabular}{ll}
\hline $\begin{array}{l}62 \\
\text { (Norway) }\end{array}$ & $\begin{array}{l}\text { To analyze the relationships between depressive symp- } \\
\text { toms, SOC and emotional and social loneliness }\end{array}$ \\
\hline 68 & $\begin{array}{l}\text { To study the effect of stressful life events on depressive } \\
\text { symptoms in elderly with extreme internal LOC com- } \\
\text { (USA) }\end{array}$ \\
& pared to those with moderate levels of internal LOC
\end{tabular}

227 older patients from a nursing home setting $\mathrm{MA}=85.4$ years, range $65-102$ years, $72 \%$ females

351 older community residents including senior citizen

GDS

housing, metropolitan area, from random survey

$\mathrm{MA}=73.4$ years, $\mathrm{SD}=6.2$ years, $66 \%$ females
CES-D pared to those with moderate levels of internal LOC

\begin{tabular}{ll}
\hline $\begin{array}{l}69 \\
\text { (Poland) }\end{array}$ & $\begin{array}{l}\text { To determine the connection between the SOC level and } \\
\text { sociodemographic factors }\end{array}$ \\
\hline 74 & $\begin{array}{l}\text { To examine whether religious coping is inversely } \\
\text { related to all depressive symptoms or only to certain } \\
\text { symptoms }\end{array}$ \\
\hline 75 & $\begin{array}{l}\text { To examine the impact of stressful life events on change } \\
\text { (Hong }\end{array}$ \\
Kong) &
\end{tabular}

257 older community residents being students of the $\mathrm{U}^{3} \mathrm{~A}^{1}$

GDS-15 $\mathrm{MA}=64.54$ years, $2 \%$ females

832 older patients from a somatic health care setting with Ham-D medical and neurological disorders SADS $\mathrm{MA}=70$ years, $0 \%$ females

GDS

411 older community residents, randomly selected from CES-D metropolitan area

$\mathrm{MA}=71.1$ years, $54.3 \%$ females

\begin{tabular}{lll}
\hline $\begin{array}{l}76 \\
\text { (USA) }\end{array}$ & $\begin{array}{l}\text { To study if LOC mediate the effect of discrimination on } \\
\text { depressive symptoms }\end{array}$ & $\begin{array}{l}\text { 472 older community residents and Korean American } \\
\text { immigrants } \\
\text { MA = 69.9 years, SD = 7.04 years, 57.9\% females }\end{array}$ \\
\hline $\begin{array}{lll}77 \\
(\text { New Zealand) }\end{array}$ & $\begin{array}{l}\text { To examine the influence of experiential avoidance on } \\
\text { depression and as a moderator in the relation between } \\
\text { physical health problems and depression }\end{array}$ & $\begin{array}{l}195 \text { older community residents living in their own homes or } \\
\text { in a retirement village complex } \\
\text { MA }=80-84 \text { years, } 71.8 \% \text { females }\end{array}$
\end{tabular}

78 To investigate the relation between self-efficacy and (USA) depression
GDS arthritis

MA $=81.7$ years, $88 \%$ females 


\begin{tabular}{|c|c|c|c|}
\hline $\begin{array}{l}\text { Instruments used to } \\
\text { assess coping }\end{array}$ & $\begin{array}{l}\text { Findings of relations between depression and } \\
\text { coping }\end{array}$ & Findings of no relations & Comments on method \\
\hline
\end{tabular}

\begin{tabular}{l} 
Coping strategies \\
Control orientation \\
CISS \\
Coping self-efficacy \\
\hline Coping strategies \\
Brief COPE \\
\hline Religious coping \\
Duke University religion \\
index
\end{tabular}

High degree of task-oriented and less use of emotion-oriented coping strategies together with higher self-efficacy were associated with decreased level of depressive symptoms

Less use of the coping strategies denial and selfblame were independently associated with decreased depressive symptom level

Increased religious coping was associated with increased disability in depressed elderly

\begin{tabular}{|c|c|c|c|}
\hline $\begin{array}{l}\text { Sense of coherence } \\
\text { SOC-19 }\end{array}$ & $\begin{array}{l}\text { Depressive group: } \\
\text { An interaction was found between verbal problem } \\
\text { solving and SOC } \\
\text { Higher functioning in problem solving and higher } \\
\text { SOC scores was associated with lower depressive } \\
\text { scores }\end{array}$ & $\begin{array}{l}\text { Control group: } \\
\text { No associations were found }\end{array}$ & $\begin{array}{l}\text { Nonparametric statistics Wil- } \\
\text { coxon sum rank } \\
\text { ANCOVA }\end{array}$ \\
\hline $\begin{array}{l}\text { Sense of coherence } \\
\text { SOC-13 }\end{array}$ & $\begin{array}{l}\text { SOC reduced the association between GDS and } \\
\text { attachment }\end{array}$ & $\begin{array}{l}\text { No interaction was found } \\
\text { between GDS and SOC }\end{array}$ & $\begin{array}{l}\text { Cronbach's alpha } \\
\text { Multiple regression analyses }\end{array}$ \\
\hline $\begin{array}{l}\text { Control orientation } \\
\text { I/E scale }\end{array}$ & $\begin{array}{l}\text { High mastery control beliefs were associated with } \\
\text { reduced depressive score } \\
\text { Lower belief in chance was associated with reduced } \\
\text { depressive score and the stress on depressive } \\
\text { symptoms was lower } \\
\text { Those with a low degree of life stress and belief in } \\
\text { chance had relatively less depressive symptoms } \\
\text { compared to those who did believe in chance and } \\
\text { had high life stress }\end{array}$ & & $\begin{array}{l}\text { Multiple linear regression analy- } \\
\text { sis adjusted for age, gender, } \\
\text { race, education, income, } \\
\text { response bias }\end{array}$ \\
\hline $\begin{array}{l}\text { Sense of coherence } \\
\text { SOC-29 }\end{array}$ & SOC was negatively associated with depression & & $\begin{array}{l}\text { Descriptive statistics } \\
\text { Spearman and Pearson's corre- } \\
\text { lation test }\end{array}$ \\
\hline $\begin{array}{l}\text { Religious coping } \\
\text { RCI }\end{array}$ & $\begin{array}{l}\text { A high religious coping was inversely related to } \\
\text { cognitive symptoms of depression }\end{array}$ & $\begin{array}{l}\text { A high religious coping was not } \\
\text { related to somatic symptoms of } \\
\text { depression }\end{array}$ & $\begin{array}{l}\text { Cochran-Mantel-Haenszel } \chi^{2} \\
\text { statistics adjusted for race, } \\
\text { psychiatric history and health } \\
\text { status }\end{array}$ \\
\hline $\begin{array}{l}\text { Control orientation } \\
\text { The generalized SOC }\end{array}$ & $\begin{array}{l}\text { A higher SOC was related to lower depressive } \\
\text { scores and mediated the impact of stressful life } \\
\text { events on depression }\end{array}$ & & $\begin{array}{l}\text { Multiple linear regression analy- } \\
\text { sis adjusted for age, gender, } \\
\text { marital status, years of educa- } \\
\text { tion chronic illnesses, functional } \\
\text { disability }\end{array}$ \\
\hline $\begin{array}{l}\text { Control orientation } \\
\text { Mastery scale }\end{array}$ & $\begin{array}{l}\text { Higher LOC was associated with lower depression } \\
\text { symptoms } \\
\text { LOC removed the effect of discrimination on } \\
\text { depressive symptoms }\end{array}$ & & $\begin{array}{l}\text { Multiple linear regression analy- } \\
\text { sis adjusted for age, gender, } \\
\text { education, length of stay in USA }\end{array}$ \\
\hline $\begin{array}{l}\text { Coping strategies } \\
\text { AAQ }\end{array}$ & $\begin{array}{l}\text { Reduced use of experiential avoidance was associ- } \\
\text { ated with a lower degree of depressive symptoms } \\
\text { Lower levels of experiential avoidance moderated } \\
\text { more strongly the association between poorer } \\
\text { physical health and higher depressive score }\end{array}$ & & $\begin{array}{l}\text { Multiple linear regression analy- } \\
\text { sis adjusted for degree of } \\
\text { impairment, self-reported } \\
\text { health and social support }\end{array}$ \\
\hline $\begin{array}{l}\text { Control orientation } \\
\text { Arthritis Self-Efficacy } \\
\text { scale } \\
\text { CPCI }\end{array}$ & $\begin{array}{l}\text { Higher self-efficacy was associated with lower } \\
\text { degree of depressive symptoms }\end{array}$ & & $\begin{array}{l}\text { Multiple linear regression analy- } \\
\text { sis adjusted for age, gender and } \\
\text { pain intensity }\end{array}$ \\
\hline
\end{tabular}

Avoidance-oriented coping was Multiple linear regression analynot related to depressive symp- sis adjusted for life stress toms

Adaptive coping styles and self-distraction were not associ- sis adjusted for severity of heart ated with depression scores failure and comorbid illness

Multiple linear regression analysis adjusted for level of disability, social support and number coxon sum rank Multiple regression analyse race, education, income

Descriptive statistics lation test psychiatric history and health Multiple linear regression analyMultiple linear regression analysis adjusted for age, gender, symptoms

LOC removed the effect of discrimination on

Multiple linear regression analy justed for degree of pairment, self-reported for age, gender and 
Table 3 (continued)

\begin{tabular}{|c|c|c|c|}
\hline $\begin{array}{l}\text { Ref. No. } \\
\text { (country) }\end{array}$ & Aim of the study ${ }^{1}$ & Sample characteristics & $\begin{array}{l}\text { Category }{ }^{2} \text { and } \\
\text { instruments used } \\
\text { to assess depres- } \\
\text { sion }\end{array}$ \\
\hline $\begin{array}{l}79 \\
\text { (USA) }\end{array}$ & $\begin{array}{l}\text { To study coping of chronic obstructive airway disease } \\
\text { in relation to depression in patients and spouses }\end{array}$ & $\begin{array}{l}107 \text { older community residents and married couples, where } \\
1 \text { had a chronic obstructive airway disease } \\
\text { MA }=62.4 \text { years, } S D=10.62 \text { years, } 37.4 \% \text { females } \\
\text { Spouses: } \\
\text { MA }=61.12 \text { years, } S D=10.63 \text { years, } 62.6 \% \text { females }\end{array}$ & CES-D \\
\hline
\end{tabular}

$41 \quad$ To explore coping actions taken to cope with depressed

(Netherlands) mood

350 older community residents living in residential homes DSM-IV in metropolitan area without cognitive impairment

CES-D

\begin{tabular}{|c|c|c|c|}
\hline $\begin{array}{l}81 \\
\text { (USA) }\end{array}$ & $\begin{array}{l}\text { To study if elderly with internal LOC had less depres- } \\
\text { sive symptoms than elderly with external LOC }\end{array}$ & $\begin{array}{l}117 \text { older community residents in a metropolitan area } \\
\mathrm{MA}=72.1 \text { years, } \mathrm{SD}=7.1 \text { years }\end{array}$ & $\begin{array}{l}\text { BDI } \\
\text { POMS } \\
\text { Ham-D } \\
\text { SDS }\end{array}$ \\
\hline $\begin{array}{l}35 \\
\text { (USA) }\end{array}$ & $\begin{array}{l}\text { To investigate the relation between religious motiva- } \\
\text { tion and beliefs and religious coping, and between } \\
\text { religious coping, depression and control beliefs }\end{array}$ & $\begin{array}{l}97 \text { older patients from a nursing home setting } \\
\text { MA }=69 \text { years, } 7.77 \% \text { females }\end{array}$ & $\begin{array}{l}\text { GDS } \\
\text { GRECC }\end{array}$ \\
\hline
\end{tabular}

\begin{tabular}{lll}
\hline 28 & To find out how SOC, depressive mood and social net- & 58 older community residents \\
(Sweden) & work relate to the tendency to report symptoms of & MA = 71 years, 47\% females \\
& illness
\end{tabular}

\begin{tabular}{lll}
\hline 60 & To identify factors associated with low SOC in elderly & $\begin{array}{l}80 \text { older persons from a somatic health care setting after } \\
\text { suicide attempt }\end{array}$ \\
(Sweden) & suicide attempters & MA $=79.4$ years, range 70-91 years, 42 females
\end{tabular}

\begin{tabular}{|c|c|c|c|}
\hline $\begin{array}{l}82 \\
\text { (Canada) }\end{array}$ & $\begin{array}{l}\text { To obtain more information about the relationships of } \\
\text { alcohol and medication use and other areas of the older } \\
\text { person's life }\end{array}$ & $\begin{array}{l}826 \text { older community residents } \\
\text { MA }=74.8 \text { years, range } 63-96,65 \% \text { females }\end{array}$ & CES-D \\
\hline $\begin{array}{l}83 \\
\text { (Finland) }\end{array}$ & $\begin{array}{l}\text { To locate several meaning-in-life measures within the } \\
\text { space of functioning }\end{array}$ & $\begin{array}{l}55 \text { older community residents in } 2 \text { age cohorts from a Finn- } \\
\text { ish population study } \\
67 \% \text { females }\end{array}$ & CES-D \\
\hline
\end{tabular}

\begin{tabular}{|c|c|c|c|}
\hline $\begin{array}{l}46 \\
\text { (Spain) }\end{array}$ & $\begin{array}{l}\text { To analyze how cognitive-behavioral and perceptual } \\
\text { variables help to explain the presence or absence of } \\
\text { depression }\end{array}$ & $\begin{array}{l}104 \text { older patients from a somatic health care setting from } \\
\text { hospitals and nursing homes with chronic pain from osteo- } \\
\text { arthritis } \\
\text { MA }=74.32 \text { years, } S D=12.6 \text { years, } 85.6 \% \text { females }\end{array}$ & GDS \\
\hline $\begin{array}{l}51 \\
(\mathrm{UK})\end{array}$ & $\begin{array}{l}\text { To examine the relation between perceptions of illness } \\
\text { and coping styles and depression }\end{array}$ & $\begin{array}{l}98 \text { older patients from a somatic health care setting } \\
\text { recruited at memory clinics with subjective memory com- } \\
\text { plaints } \\
\mathrm{MA}=73.4 \text { years, } \mathrm{SD}=8.8,64 \text { females }\end{array}$ & GDS-15 \\
\hline $\begin{array}{l}86 \\
(\text { USA) }\end{array}$ & $\begin{array}{l}\text { To determine the relation between religiosity, depres- } \\
\text { sion, and other mood states }\end{array}$ & $\begin{array}{l}100 \text { older patients from a somatic health care setting with } \\
\text { cancer } \\
\text { MA }=73 \text { years, } 67 \% \text { females }\end{array}$ & $\begin{array}{l}\text { GDS } \\
\text { POMS }\end{array}$ \\
\hline
\end{tabular}




\begin{tabular}{|c|c|c|c|}
\hline $\begin{array}{l}\text { Instruments used to } \\
\text { assess coping }\end{array}$ & $\begin{array}{l}\text { Findings of relations between depression and } \\
\text { coping }\end{array}$ & Findings of no relations & Comments on method \\
\hline $\begin{array}{l}\text { Coping strategies } \\
\text { Control orientation } \\
\text { Indices of coping } \\
\text { resources } \\
\text { MHLOC }\end{array}$ & $\begin{array}{l}\text { Patients: } \\
\text { High degree of active, behavioral and cognitive } \\
\text { coping and low degree of avoidance coping were } \\
\text { associated with less depressive symptoms } \\
\text { Spouses: } \\
\text { Higher internal control, less avoidant coping and } \\
\text { more active coping strategies were related to less } \\
\text { depressive symptoms }\end{array}$ & $\begin{array}{l}\text { Patients: } \\
\text { HLOC - belief was not signifi- } \\
\text { cantly related to depression }\end{array}$ & $\begin{array}{l}\text { Multiple linear regression analy } \\
\text { sis adjusted for gender and age }\end{array}$ \\
\hline $\begin{array}{l}\text { Coping strategies } \\
22 \text { coping actions, adapted } \\
\text { from the questionnaire of } \\
\text { Jorm et al., } 2004 \text { [158] }\end{array}$ & $\begin{array}{l}\text { The odds for using coping actions against depres- } \\
\text { sion decreased with low depression score and no } \\
\text { lifetime major depressive disorder }\end{array}$ & & $\begin{array}{l}\text { Multiple logistic regression } \\
\text { analysis adjusted for gender, } \\
\text { age, civil status, education } \\
\text { religious belief }\end{array}$ \\
\hline $\begin{array}{l}\text { Control orientation } \\
\text { Levenson's Internal (I), } \\
\text { Powerful Others (P), and } \\
\text { Chance (C) scales }\end{array}$ & $\begin{array}{l}\text { Higher internality and lower belief in powerful } \\
\text { others were associated with lower depression score }\end{array}$ & $\begin{array}{l}\text { Higher belief in chance was not } \\
\text { associated with high or low } \\
\text { depressive scores }\end{array}$ & $\begin{array}{l}\text { Pearson's correlation } \\
\text { One-way ANOVA }\end{array}$ \\
\hline $\begin{array}{l}\text { Control orientation } \\
\text { Religious coping } \\
\text { I/E-R scale } \\
\text { RCI } \\
\text { RPSS } \\
\text { HLOC }\end{array}$ & $\begin{array}{l}\text { Increased religious coping was related to increased } \\
\text { control beliefs and higher control beliefs were } \\
\text { associated with reduced depression score }\end{array}$ & & $\begin{array}{l}\text { Multiple linear regression analy- } \\
\text { sis }\end{array}$ \\
\hline $\begin{array}{l}\text { Sense of coherence } \\
\text { SOC-29 }\end{array}$ & $\begin{array}{l}\text { Higher SOC scores correlated with less depressive } \\
\text { symptoms }\end{array}$ & & $\begin{array}{l}\text { Pearson's correlation } \\
\text { Multivariate analyses }\end{array}$ \\
\hline $\begin{array}{l}\text { Sense of coherence } \\
\text { SOC- } 29\end{array}$ & $\begin{array}{l}\text { Major depression was strongly and positively asso- } \\
\text { ciated with SOC }\end{array}$ & & $\begin{array}{l}\text { Univariate and multivariate } \\
\text { exact logistic regression } \\
\text { adjusted for sex, age, current } \\
\text { major depression }\end{array}$ \\
\hline $\begin{array}{l}\text { Sense of coherence } \\
\text { SOC-13 }\end{array}$ & $\begin{array}{l}\text { Depression was highest, and SOC lowest for former } \\
\text { drinkers, with no significant differences among the } \\
4 \text { groups }\end{array}$ & & Analyses of covariance \\
\hline $\begin{array}{l}\text { Sense of coherence } \\
\text { SOC-13 }\end{array}$ & $\begin{array}{l}\text { Higher SOC score was associated with lower } \\
\text { depressive symptoms in persons who see their life } \\
\text { as a rising line }\end{array}$ & & $\begin{array}{l}\text { Multidimensional scaling in a } \\
\text { distance matrix } \\
\text { Kruskal's stress formula } \\
\text { Squared correlations } \\
\text { Pearson's correlation }\end{array}$ \\
\hline $\begin{array}{l}\text { Coping strategies } \\
\text { CSQ } \\
\text { Vanderbilt pain manage- } \\
\text { ment }\end{array}$ & $\begin{array}{l}\text { A lower degree of complaint behavior, avoidance, } \\
\text { catastrophizing, passive coping style, stability } \\
\text { beliefs and mystery beliefs and higher degree of } \\
\text { ignoring pain sensations and coping self-statements } \\
\text { were related to absence of depression }\end{array}$ & $\begin{array}{l}\text { No differences were found } \\
\text { between those with and with- } \\
\text { out symptoms of depression for } \\
\text { active coping, self-blame, self- } \\
\text { efficacy for controlling pain and } \\
\text { for decreasing pain, reinterpret- } \\
\text { ing pain sensations, praying and } \\
\text { hoping, diverting attention and } \\
\text { help-seeking }\end{array}$ & 2-group comparison with t tests \\
\hline $\begin{array}{l}\text { Coping strategies } \\
\text { Control orientation } \\
\text { WCQ } \\
\text { Personal control belief }\end{array}$ & $\begin{array}{l}\text { Greater use of escape/avoidance and less use of } \\
\text { distancing correlated with higher depression score } \\
\text { Higher personal control was associated with lower } \\
\text { depression score }\end{array}$ & $\begin{array}{l}\text { Avoidance and distancing were } \\
\text { not significantly associated with } \\
\text { depression }\end{array}$ & $\begin{array}{l}\text { Pearson's correlation analysis } \\
\text { and multiple linear regression } \\
\text { analysis adjusted for perception } \\
\text { of illness }\end{array}$ \\
\hline $\begin{array}{l}\text { Religious coping } \\
\text { (I/E-R) scale }\end{array}$ & $\begin{array}{l}\text { High intrinsic religiosity patients had lower depres- } \\
\text { sion scores and better mood state than those with } \\
\text { low intrinsic religiosity }\end{array}$ & & ANCOVA \\
\hline
\end{tabular}


Table 3 (continued)

\begin{tabular}{|c|c|c|c|}
\hline $\begin{array}{l}\text { Ref. No. } \\
\text { (country) }\end{array}$ & Aim of the study ${ }^{1}$ & Sample characteristics & $\begin{array}{l}\text { Categor } \\
\text { instrum } \\
\text { to asses } \\
\text { sion }\end{array}$ \\
\hline $\begin{array}{l}87 \\
\text { (Netherlands) }\end{array}$ & $\begin{array}{l}\text { To study the relation between coping in terms of LOC, } \\
\text { cognitive function and depressive symptoms }\end{array}$ & $\begin{array}{l}\text { 3,107 older community residents, randomly selected from } 9 \\
\text { municipalities } \\
\mathrm{MA}=70.8 \text { years, } \mathrm{SD}=8.8 \text { years, } 51.5 \% \text { females }\end{array}$ & CES-D \\
\hline $\begin{array}{l}88 \\
(\mathrm{UK})\end{array}$ & $\begin{array}{l}\text { To examine health LOC in relation to depressive symp- } \\
\text { toms }\end{array}$ & $\begin{array}{l}1,602 \text { older patients from a somatic health care setting } \\
\text { recruited from } 2 \mathrm{GPs} \\
>65 \text { years, } 62.5 \% \text { females }\end{array}$ & GDS- \\
\hline
\end{tabular}

$>65$ years, $62.5 \%$ females

\begin{tabular}{lll}
\hline 89 & To investigate LOC as risk factor for depressive symp- & $\begin{array}{l}1,116 \text { older community residents, randomly selected in a } \\
\text { (Spain) }\end{array}$ \\
toms among an elderly Spanish population & $>65$ years, $45.9 \%$ females
\end{tabular}

Studies of less than 6 quality criteria

$90 \quad$ To study the relation between internal health LOC and 508 older community residents

(USA) depression by gender

MA $=72.5$ years females, 71.1 years males, $58.9 \%$ females

18-item scale of depressive sys-

tems

\begin{tabular}{lll}
\hline 61 & To study dispositional coping styles and distress among & 28 older patients from a somatic health care setting with \\
(USA) & cognitively impaired anxious/depressed and nonde- & cognitive impairment in a memory clinic \\
pressed persons & MA $=79.5$ years, SD $=7.7$ years, 82\% females \\
& 42 anxious/depressed patients without cognitive impair- \\
& ment \\
& MA $=64.4$ years, SD $=6.8$ years, $64 \%$ females \\
& 25 healthy older control group participants \\
& MA $=63.7$ years, SD = 7.1 years, 56\% females
\end{tabular}

$\begin{array}{ll}94 & \text { To study whether or not the relation between external } \\ \text { (USA) } & \text { LOC orientation and depression correlate }\end{array}$

23 older community residents without depression and 25

SDS with depression

Range 60-80 years

$95 \quad$ To examine the effect of SOC in the relation between

(Hong financial strain and depressive symptoms

411 older community residents, randomly selected from

CES-D

Kong)

$\mathrm{MA}=71.1$ years, $54.3 \%$ females

104 older patients from a somatic health care setting being
veterans with heart failure $\mathrm{MA}=71.7$ years, $1 \%$ females
GDS

(USA) depressive symptoms struments used assess depres- 
Bjørkløf et al.: Coping and Depression in Old Age: A Literature Review

\begin{tabular}{|c|c|c|c|}
\hline $\begin{array}{l}\text { Instruments used to } \\
\text { assess coping }\end{array}$ & $\begin{array}{l}\text { Findings of relations between depression and } \\
\text { coping }\end{array}$ & Findings of no relations & Comments on method \\
\hline $\begin{array}{l}\text { Control orientation } \\
\text { Mastery scale }\end{array}$ & $\begin{array}{l}\text { Women, but not men, with high cognitive function } \\
\text { and an internal control had less depressive symp- } \\
\text { toms compared to low cognitive functioning woman } \\
\text { with an external LOC }\end{array}$ & & $\begin{array}{l}\text { Multiple linear regression analy- } \\
\text { sis adjusted for age and educa- } \\
\text { tion }\end{array}$ \\
\hline $\begin{array}{l}\text { Control orientation } \\
\text { HLOC }\end{array}$ & $\begin{array}{l}\text { High degree of internality and low degree of belief } \\
\text { in chance were both associated with reduced odds } \\
\text { for depression }\end{array}$ & & $\begin{array}{l}\text { Multiple logistic regression } \\
\text { analysis adjusted for age, gen- } \\
\text { der and general practice, and } \\
\text { several health and disability } \\
\text { variables }\end{array}$ \\
\hline $\begin{array}{l}\text { Control orientation } \\
\text { Mastery scale }\end{array}$ & $\begin{array}{l}\text { High LOC was associated with decreased risk of } \\
\text { depression }\end{array}$ & & $\begin{array}{l}\text { Multiple logistic regression } \\
\text { analysis adjusted for gender, } \\
\text { income, chronic condition ADL } \\
\text { and IADL impairment, social } \\
\text { support, and social activities }\end{array}$ \\
\hline $\begin{array}{l}\text { Control orientation } \\
\text { MHLC }\end{array}$ & $\begin{array}{l}\text { Higher internal health LOC was associated with less } \\
\text { depressive symptoms for women, but not for men }\end{array}$ & & $\begin{array}{l}\text { Regression analysis adjusting } \\
\text { for age }\end{array}$ \\
\hline $\begin{array}{l}\text { Coping strategies } \\
\text { COPE }\end{array}$ & $\begin{array}{l}\text { The group of persons with cognitive impairment } \\
\text { had less use of problem-focused and emotional- } \\
\text { focused coping strategies than the group with } \\
\text { anxiety and depression, and did not differ from the } \\
\text { control group }\end{array}$ & & $\begin{array}{l}\text { 3-group comparison with use of } \\
\text { Anova and Bonferroni posttests }\end{array}$ \\
\hline $\begin{array}{l}\text { Control orientation } \\
\text { I/E scale }\end{array}$ & $\begin{array}{l}\text { Internal LOC correlates with less depressive symp- } \\
\text { toms }\end{array}$ & & $\begin{array}{l}\text { Pearson's correlation } \\
\text { 2-group comparison }\end{array}$ \\
\hline $\begin{array}{l}\text { Control orientation } \\
\text { SOC over finances } \\
\text { The generalized SOC }\end{array}$ & $\begin{array}{l}\text { Higher degree of generalized SOC was significantly } \\
\text { associated with lower depressive symptoms } \\
\text { When level of generalized SOC became higher, the } \\
\text { impact of financial strain on depressive symptoms } \\
\text { was lower }\end{array}$ & $\begin{array}{l}\text { SOC over finances was not } \\
\text { associated with depressive } \\
\text { symptoms }\end{array}$ & $\begin{array}{l}\text { Multiple linear regression analy- } \\
\text { sis adjusted for age, gender, } \\
\text { marital status, years of educa- } \\
\text { tion and financial strain }\end{array}$ \\
\hline $\begin{array}{l}\text { Control orientation } \\
\text { Coping strategies } \\
\text { MHLC } \\
\text { Brief-COPE }\end{array}$ & $\begin{array}{l}\text { Higher self-efficacy, less attributed LOC to chance } \\
\text { and less maladaptive coping responses were related } \\
\text { to less depression } \\
\text { Attributing LOC to chance independently predicted } \\
\text { depressive symptoms }\end{array}$ & $\begin{array}{l}\text { Depressed did not significantly } \\
\text { differ from nondepressed in } \\
\text { terms of adaptive coping, reli- } \\
\text { gious/substance coping and } \\
\text { internal LOC }\end{array}$ & $\begin{array}{l}\text { 2-group comparison } \\
\text { Multiple linear regression analy- } \\
\text { sis adjusted for physical limita- } \\
\text { tion, heart failure intrusiveness, } \\
\text { maladaptive coping and self- } \\
\text { efficacy }\end{array}$ \\
\hline $\begin{array}{l}\text { Coping strategies } \\
\text { Dispositional Resilience } \\
\text { scale II }\end{array}$ & Hardiness and depression correlated & & Pearson's correlation \\
\hline $\begin{array}{l}\text { Control orientation } \\
\text { MHLC }\end{array}$ & $\begin{array}{l}\text { High belief in internal health LOC correlated with } \\
\text { and was associated with low depression score }\end{array}$ & $\begin{array}{l}\text { A belief in external and chance } \\
\text { health LOC were not correlated } \\
\text { with depression score }\end{array}$ & $\begin{array}{l}\text { Pearson's product/moment } \\
\text { correlation } \\
\text { Multiple linear regression analy- } \\
\text { sis adjusted for cognitive status, } \\
\text { heritage culture and physical } \\
\text { health }\end{array}$ \\
\hline $\begin{array}{l}\text { Coping strategies } \\
\text { Goal adjustment scale }\end{array}$ & $\begin{array}{l}\text { Low score on negative affect and high score on } \\
\text { positive affect correlated with lower degree of } \\
\text { depressive symptoms }\end{array}$ & & $\begin{array}{l}\text { Logistic regression analysis } \\
\text { Structural equation modeling } \\
\text { Correlation analysis (not told } \\
\text { whether it was nonparametric } \\
\text { or parametric) }\end{array}$ \\
\hline
\end{tabular}


Table 3 (continued)

\begin{tabular}{|c|c|c|c|}
\hline $\begin{array}{l}\text { Ref. No. } \\
\text { (country) }\end{array}$ & Aim of the study ${ }^{1}$ & Sample characteristics & $\begin{array}{l}\text { Category }{ }^{2} \text { and } \\
\text { instruments used } \\
\text { to assess depres- } \\
\text { sion }\end{array}$ \\
\hline $\begin{array}{l}99 \\
\text { (Sweden) }\end{array}$ & $\begin{array}{l}\text { To investigate the correlations between measures of } \\
\text { dispositional optimism and depression }\end{array}$ & $\begin{array}{l}68 \text { older patients from a somatic health care setting with } \\
\text { hearing impairments from register at local hearing center } \\
\mathrm{MA}=69.6 \text { years, } \mathrm{SD}=2.5 \text { years, } 44.1 \% \text { females }\end{array}$ & BDI \\
\hline
\end{tabular}

\begin{tabular}{lll}
\hline 36 & To examine spiritual well-being and practice of patients & $\begin{array}{l}\text { 24 older patients from a somatic health care setting with a } \\
\text { primary diagnosis of severe heart failure }\end{array}$ \\
in relation to depression & MA $=76.2$ years, SD $=9.29$ years, $10 \%$ females
\end{tabular}

CES-D $\mathrm{MA}=76.2$ years, $\mathrm{SD}=9.29$ years, $10 \%$ females

$\begin{array}{ll}42 & \text { To examine the potential relations between spirituality } \\ \text { (USA) } & \text { and depression }\end{array}$

\begin{tabular}{lll}
\hline 100 & To examine the influence of lifetime-accumulated & 1,216 older community residents \\
(USA) & trauma on mental health & MA $=74.2$ years, $67 \%$ females
\end{tabular}

\begin{tabular}{ll}
\hline $\begin{array}{l}\text { (China) } \\
\text { To evaluate the impact of coping and other factors on } \\
\text { depression in empty-nest elderly }\end{array}$ \\
\hline 102 & $\begin{array}{l}\text { To examine the association between depressive symp- } \\
\text { toms and health (physically, functionally and mentally) }\end{array}$
\end{tabular}

415 older community residents, empty nest and nonempty

$\begin{array}{ll}103 & \text { To examine the relationship between illness percep- } \\ \text { (UK) } & \text { tions, adaptive coping and depression }\end{array}$

104 (Netherlands) related to depressive symptoms
301 older patients from a nursing home setting, recruited from palliative care service $\mathrm{MA}=68.5$ years, $49 \%$ females
Primary Care Evaluation of Mental Disorders (PRIME-MD)

\begin{tabular}{|c|c|c|c|}
\hline $\begin{array}{l}105 \\
\text { (USA) }\end{array}$ & $\begin{array}{l}\text { To evaluate whether elderly with depression use differ- } \\
\text { ent coping strategies than elderly without depression } \\
\text { and if these strategies were perceived to be helpful } \\
\text { regarding a negative life event }\end{array}$ & $\begin{array}{l}32 \text { older patients from a psychogeriatric clinical setting with } \\
\text { major depression } \\
\mathrm{MA}=71.9 \text { years, } \mathrm{SD}=4.73 \text { years, } 81.25 \% \text { females } \\
32 \text { older community residents from local senior service } \\
\text { centers as control group } \\
\mathrm{MA}=73.2 \text { years, } \mathrm{SD}=6.29 \text { years, } 81.25 \% \text { females }\end{array}$ & BDI \\
\hline $\begin{array}{l}48 \\
\text { (USA) }\end{array}$ & $\begin{array}{l}\text { To study if behavioral and approach coping strategies } \\
\text { would be associated with less depressive symptoms }\end{array}$ & $\begin{array}{l}80 \text { older patients from a somatic health care setting with } \\
\text { Parkinson's disease } \\
\text { MA }=61.7 \text { years, } 31.3 \% \text { females }\end{array}$ & BDI \\
\hline $\begin{array}{l}106 \\
\text { (Canada) }\end{array}$ & $\begin{array}{l}\text { To examine the use of proactive coping in relation to } \\
\text { depression }\end{array}$ & $\begin{array}{l}224 \text { older community residents in day program at commu- } \\
\text { nity centers } \\
\text { MA }=75 \text { years, } S D=6.88 \text { years, } 78.2 \% \text { females }\end{array}$ & BSI \\
\hline
\end{tabular}




\begin{tabular}{|c|c|c|c|}
\hline $\begin{array}{l}\text { Instruments used to } \\
\text { assess coping }\end{array}$ & $\begin{array}{l}\text { Findings of relations between depression and } \\
\text { coping }\end{array}$ & Findings of no relations & Comments on method \\
\hline $\begin{array}{l}\text { Control orientation } \\
\text { LOT } \\
\text { HCA }\end{array}$ & $\begin{array}{l}\text { A high degree of optimism (Life Orientation test) } \\
\text { correlated with a low depression score }\end{array}$ & & $\begin{array}{l}\text { Cronbach's alpha } \\
\text { Pearson's correlation } \\
\text { One-way ANOVA }\end{array}$ \\
\hline $\begin{array}{l}\text { Control orientation } \\
\text { Religious coping } \\
\text { SIWB (self-efficacy }+ \text { life } \\
\text { scheme subscales) } \\
\text { SPC }\end{array}$ & $\begin{array}{l}\text { A higher self-efficacy correlated strongly with a } \\
\text { decrease in depressive symptoms } \\
\text { As spiritual well-being increased, depression } \\
\text { decreased }\end{array}$ & & Pearson's correlation \\
\hline $\begin{array}{l}\text { Religious coping } \\
\text { DSES }\end{array}$ & $\begin{array}{l}\text { Higher religious coping correlated moderately with } \\
\text { lower depression score } \\
\text { Religiosity tended to be associated with reduced } \\
\text { depressive symptom scores }\end{array}$ & & $\begin{array}{l}\text { Pearson's correlation } \\
\text { Multiple linear regression analy- } \\
\text { sis adjusted for age, gender, } \\
\text { race, income, home service } \\
\text { support, loneliness }\end{array}$ \\
\hline $\begin{array}{l}\text { Coping strategies } \\
\text { PTSD checklist }\end{array}$ & $\begin{array}{l}\text { Reduced avoidance behavior is associated with } \\
\text { reduced depressive score and acts as a moderator } \\
\text { of the association between higher accumulated } \\
\text { trauma and increased depressive score }\end{array}$ & & $\begin{array}{l}\text { Multiple linear regression analy- } \\
\text { sis adjusted for age, gender and } \\
\text { civil status }\end{array}$ \\
\hline $\begin{array}{l}\text { Coping strategies } \\
\text { CISS }\end{array}$ & $\begin{array}{l}\text { Use of more emotion- and avoidance-oriented } \\
\text { strategies and less adaptive coping (i.e. task-ori- } \\
\text { ented) dominated in those with depression com- } \\
\text { pared to those without depression }\end{array}$ & & $\begin{array}{l}\text { Univariate linear regression } \\
\text { analysis was performed con- } \\
\text { cerning religious activity, but } \\
\text { not the CISS } \\
\chi^{2} \text { analysis }\end{array}$ \\
\hline $\begin{array}{l}\text { Coping strategies } \\
\text { SCSQ }\end{array}$ & $\begin{array}{l}\text { Having a low negative coping style was indepen- } \\
\text { dently associated with reduced depressive score }\end{array}$ & $\begin{array}{l}\text { Positive coping style was not } \\
\text { associated with depressive } \\
\text { score }\end{array}$ & $\begin{array}{l}\text { Multiple linear regression analy- } \\
\text { sis adjusted for support, marital } \\
\text { status and economic status }\end{array}$ \\
\hline $\begin{array}{l}\text { Control orientation } \\
\text { HLOC }\end{array}$ & $\begin{array}{l}\text { Higher experienced control over health reduced the } \\
\text { risk for depression in women, but not in men }\end{array}$ & & $\begin{array}{l}\text { Multiple logistic regression } \\
\text { analysis adjusted for education, } \\
\text { insurance coverage, low assimi- } \\
\text { lation }\end{array}$ \\
\hline $\begin{array}{l}\text { Coping strategies } \\
\text { Revised Coping question- } \\
\text { naire based on mental } \\
\text { adjustment to cancer scale } \\
\text { (MAC) }\end{array}$ & $\begin{array}{l}\text { Higher helplessness/hopelessness, and anxiety and } \\
\text { lower fighting spirit were correlated with higher } \\
\text { depression score }\end{array}$ & $\begin{array}{l}\text { Fatalism was not correlated } \\
\text { with depressive symptoms }\end{array}$ & $\begin{array}{l}\text { Spearman's rank correlation } \\
\text { Logistic regression analysis }\end{array}$ \\
\hline $\begin{array}{l}\text { Control orientation } \\
\text { Coping strategies } \\
\text { CLOC } \\
\text { UCL }\end{array}$ & $\begin{array}{l}\text { Low avoidance coping correlated with low depres- } \\
\text { sive scores }\end{array}$ & $\begin{array}{l}\text { Other coping styles and CLOC } \\
\text { orientations were not related to } \\
\text { depressive symptoms }\end{array}$ & $\begin{array}{l}\text { Cross-sectional correlations } \\
\text { both at baseline and follow-up }\end{array}$ \\
\hline $\begin{array}{l}\text { Coping strategies } \\
\text { HDLQ }\end{array}$ & $\begin{array}{l}\text { Nondepressed elderly used emotional discharge } \\
\text { strategies, logical analysis, information seeking, } \\
\text { problem solving and affective regulation less often } \\
\text { than the depressed } \\
\text { All coping strategies were more efficient in dealing } \\
\text { with a stressor among the nondepressed }\end{array}$ & $\begin{array}{l}\text { Nondepressed did not differ sig- } \\
\text { nificantly from depressed } \\
\text { elders regarding frequency of } \\
\text { use of logical analysis, informa- } \\
\text { tion seeking, problem-solving, } \\
\text { or affective regulation }\end{array}$ & 2-group comparison \\
\hline $\begin{array}{l}\text { Coping strategies } \\
\text { CRI }\end{array}$ & $\begin{array}{l}\text { Higher degree of behavioral coping strategies and } \\
\text { lower degree of cognitive coping strategies were } \\
\text { independently associated with lower levels of } \\
\text { depressive symptoms }\end{array}$ & $\begin{array}{l}\text { Cognitive, approach and avoid- } \\
\text { ance coping were not associ- } \\
\text { ated with depressive score }\end{array}$ & $\begin{array}{l}\text { Hierarchical linear regression } \\
\text { analysis }\end{array}$ \\
\hline $\begin{array}{l}\text { Coping strategies } \\
\text { PCI }\end{array}$ & $\begin{array}{l}\text { High degree of proactive coping is related to lower } \\
\text { depressive symptoms }\end{array}$ & & Path analysis \\
\hline
\end{tabular}


Dementia

Cognitive Disorders
Dement Geriatr Cogn Disord 2013;35:121-154

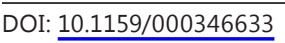

Bjørkløf et al.: Coping and Depression in Old Age: A Literature Review

C 2013 S. Karger AG, Base

www.karger.com/dem

Table 3 (continued)

\begin{tabular}{|c|c|c|c|}
\hline $\begin{array}{l}\text { Ref. No. } \\
\text { (country) }\end{array}$ & Aim of the study ${ }^{1}$ & Sample characteristics & $\begin{array}{l}\text { Category }{ }^{2} \text { and } \\
\text { instruments used } \\
\text { to assess depres- } \\
\text { sion }\end{array}$ \\
\hline $\begin{array}{l}107 \\
\text { (Canada) }\end{array}$ & $\begin{array}{l}\text { To examine the importance of coping responses in } \\
\text { relation to depressive symptoms }\end{array}$ & $\begin{array}{l}45 \text { older patients from a somatic health care setting with } \\
\text { Parkinson's disease } \\
\text { MA = } 67.4 \text { years, } 51.1 \% \text { females } \\
24 \text { older patients with comparable disabilities as control } \\
\text { group } \\
\text { MA }=65.4 \text { years, } 58 \% \text { females }\end{array}$ & BDI \\
\hline
\end{tabular}

\begin{tabular}{|c|c|c|c|}
\hline $\begin{array}{l}108 \\
\text { (USA) }\end{array}$ & $\begin{array}{l}\text { To examine if coping has main effects on the level of } \\
\text { occurrence of depressive symptoms }\end{array}$ & $\begin{array}{l}96 \text { older community residents from metropolitan areas } \\
\mathrm{MA}=74.4 \text { years, } \mathrm{SD}=6.28 \text { years, } 69.7 \% \text { females }\end{array}$ & $\begin{array}{l}\text { Health and Daily } \\
\text { Living Form }\end{array}$ \\
\hline $\begin{array}{l}109 \\
\text { (USA) }\end{array}$ & To identify if group-specific differences of coping exist & $\begin{array}{l}4,017 \text { older community residents at risk of hospitalization } \\
\geq 65 \text { years, } 100 \% \text { females }\end{array}$ & $\begin{array}{l}\text { Self-reported } \\
\text { depressive symp- } \\
\text { toms }\end{array}$ \\
\hline
\end{tabular}

\begin{tabular}{lll}
\hline 110 & To study risk factors of comorbid depression & $\begin{array}{l}216 \text { older community residents, randomly selected elderly } \\
\text { with type } 2 \text { diabetes mellitus }\end{array}$
\end{tabular}

Range $55-85$ years, $53 \%$ females

\begin{tabular}{|c|c|c|c|}
\hline $\begin{array}{l}112 \\
\text { (Canada) }\end{array}$ & $\begin{array}{l}\text { To study the correlation between desired control and } \\
\text { depressive score }\end{array}$ & $\begin{array}{l}\text { Study 1: } \\
88 \text { older community residents } \\
\text { MA = } 75 \text { years, } 75 \% \text { females } \\
\text { Study } 2 \text { : } \\
77 \text { older patients from a nursing home setting being armed } \\
\text { forces veterans in chronic care hospital ward } \\
\text { MA = } 72 \text { years, } 0 \% \text { females }\end{array}$ & $\begin{array}{l}\text { Depressive vari- } \\
\text { ables adapted } \\
\text { from Jackson and } \\
\text { Messick's Differen- } \\
\text { tial Personality } \\
\text { Inventory } \\
\text { Chiriboga's Posi- } \\
\text { tive/Negative } \\
\text { Mood Measure }\end{array}$ \\
\hline
\end{tabular}

depressive symptoms in depressed persons but not in the control group [59], and another study found SOC to mediate the association between depressive symptoms and attachment [62] (tables 2, 3).

\section{Control Orientation}

Among the total of 36 studies related to the concept of control orientation, 22 were of higher quality (10 longitudinal and 12 cross-sectional; table 1 ). In studies of higher quality, the samples were recruited from the community in 13 studies. Of the 9 studies from the clinical settings, 3 included participants with different cardiac disorders, 2 included participants suffering from depression, 2 had participants from nursing homes, 1 study had participants with cognitive decline and 1 study included participants with somatic disorders in general (GP; tables 2, 3). Among the 10 longitudinal studies of higher quality, a high 'internally oriented recovery LOC' or 'desired LOC' (perceived control in a situation), self-efficacy, optimism, mastery and a low externally oriented LOC at baseline were all associated with fewer depressive symptoms and/or less persistent depression at follow-up (table 2). Of the 
Dementia

Cognitive Disorders
Dement Geriatr Cogn Disord 2013;35:121-154

DOI: $10.1159 / 000346633$

Bjørkløf et al.: Coping and Depression in Old Age: A Literature Review

\begin{tabular}{|c|c|c|c|}
\hline $\begin{array}{l}\text { Instruments used to } \\
\text { assess coping }\end{array}$ & $\begin{array}{l}\text { Findings of relations between depression and } \\
\text { coping }\end{array}$ & Findings of no relations & Comments on method \\
\hline $\begin{array}{l}\text { Coping strategies } \\
\text { Billings and Moos Coping } \\
\text { questionnaire }\end{array}$ & $\begin{array}{l}\text { Patients: } \\
\text { Higher use of active cognitive coping strategies } \\
\text { correlated with less depressive symptoms } \\
\text { Controls: } \\
\text { Higher active behavioral coping correlated with less } \\
\text { depressive symptoms } \\
\text { Patients had higher depressive score than controls } \\
\text { and used active (cognitive and behavioral) coping } \\
\text { strategies less often }\end{array}$ & $\begin{array}{l}\text { Avoidance coping was not } \\
\text { related to depression scores in } \\
\text { either of the } 2 \text { groups }\end{array}$ & $\begin{array}{l}\text { 2-group comparison with t tests } \\
\text { Pearson's correlation }\end{array}$ \\
\hline $\begin{array}{l}\text { Coping strategies } \\
\text { COPE }\end{array}$ & $\begin{array}{l}\text { Active coping and reduced ventilation were inde- } \\
\text { pendently associated with reduced depressive } \\
\text { symptoms }\end{array}$ & $\begin{array}{l}\text { Avoidant coping and cognitive } \\
\text { coping were not independently } \\
\text { related to depressive symptoms }\end{array}$ & $\begin{array}{l}\text { Multiple linear regression analy- } \\
\text { sis adjusted for negative mood } \\
\text { and hassles occurrence }\end{array}$ \\
\hline $\begin{array}{l}\text { Control orientation } \\
\text { Coping resources }\end{array}$ & $\begin{array}{l}\text { Higher SOC in life was associated with decreased } \\
\text { depressive score in all ethnic groups of elderly }\end{array}$ & & $\begin{array}{l}\text { Multiple linear regression analy- } \\
\text { sis adjusted for age, income, } \\
\text { living alone, ADL and IADL } \\
\text { impairment, medical illness, } \\
\text { involuntary relocation and } \\
\text { social network }\end{array}$ \\
\hline $\begin{array}{l}\text { Control orientation } \\
\text { Mastery scale }\end{array}$ & $\begin{array}{l}\text { Having a higher internal LOC (mastery) was associ- } \\
\text { ated with less depressive symptoms }\end{array}$ & & $\begin{array}{l}\text { Multiple linear regression analy- } \\
\text { sis adjusted for age, civil status, } \\
\text { education clinical characteris- } \\
\text { tics, functional limitations and } \\
\text { support received }\end{array}$ \\
\hline $\begin{array}{l}\text { Control orientation } \\
\text { The Desired Control } \\
\text { questionnaire }\end{array}$ & $\begin{array}{l}\text { Study 1: } \\
\text { Higher desired control correlates with lower } \\
\text { depressive score } \\
\text { Study 2: } \\
\text { Desired control significantly correlated with fre- } \\
\text { quency of positive mood }\end{array}$ & $\begin{array}{l}\text { Study 2: } \\
\text { Desired control was not signifi- } \\
\text { cantly correlated with negative } \\
\text { mood }\end{array}$ & $\begin{array}{l}\text { Correlation } \\
\text { Cronbach's alpha }\end{array}$ \\
\hline
\end{tabular}

\footnotetext{
${ }^{1}$ Aim of the published studies was in relation to the purpose of this review study.

${ }^{2}$ The 4 main categories of coping: coping strategies (also including style and actions), control orientation, sense of coherence and religious coping.
}

12 cross-sectional studies of higher quality, a high internal LOC, self-efficacy and low externally oriented control was found to be associated with less depressive symptoms (table 3 ). Two longitudinal studies report some results of no association, i.e. 1 study including LOC and self-efficacy [63] and another including optimism [64] at baseline did not find these concepts of control orientation associated with reduced depressive symptoms after treatment (table 2). Furthermore, 2 cross-sectional studies focusing on 'belief of chance' as control orientation did not find associations with degree of depressive score (table 3).

\section{Coping Strategies}

Of the total of 37 studies concerning coping strategies in relation to depression, 14 were of higher quality (7 longitudinal and 7 cross-sectional; table 1). Nine studies had recruited older persons from clinical settings and 5 of the studies were community based. From clinical settings, 4 studies included participants with different cardiac disorders, 3 included depressed participants, 1 included participants suffering from pain, and 1 study included cognitively impaired older persons (tables 2, 3). Of the 7 longitudinal studies of higher quality, an 
Table 4. Various instruments used in screening depressive symptoms

\begin{tabular}{ll}
\hline Measurement & Number of studies \\
\hline CES-D (31) & $24(36,41,67,68,70,72,73,75,76,79,80,82,83$, \\
& $87,89,95-98,101,102,104,109,110)$ \\
GDS $(115,116)$ & $23(25,35,40,42-44,46,52,53,58,59,62,65$, \\
& $66,69,74,77,78,84,86,88,100,114)$ \\
BDI (117) & $8(48,55,57,71,81,92,99,105,107)$ \\
Ham-D (118) & $6(25,28,50,55,66,74,81)$ \\
SDS (119) & $4(81,94)$ \\
POMS (120) & $3(81,85,86)$ \\
BSI (121) & $2(61,106)$ \\
The depressive symptoms measure (122) & $2(91,108)$ \\
MADRS (123) & $2(26,56)$ \\
HADS (124) & $2(45,131)$ \\
SDS (125) & $1(90)$ \\
GRECC (126) & $1(35)$ \\
RDC (127) & $1(91)$ \\
WDI (128) & $1(92)$ \\
SADS (129) & $1(74)$ \\
'Depression' (112) & $1(112)$ \\
Chiriboga's Positive/Negative Mood measure (112) & $1(112)$ \\
Mood scale (111) & $1(111)$ \\
'Staff rating of depression' (93) & $1(93)$ \\
PRIME-MD (130) & $1(109)$ \\
\hline
\end{tabular}

Assessments of depression: GDS = Geriatric Depression Scale; GDS-SF = Geriatric Depression Scale-Short form; POMS = The Profile of Mood States; GRECC = The Durham Depression Scale; SADS = Schedule for Affective Disorder and Schizophrenia; CES-D = The Center for Epidemiologic Studies of Depression Scale; BSI = Brief Symptom Inventory; BDI = Beck Depression Inventory; SDS = Self-Rating Depression Scale; Ham$\mathrm{D}$ = Hamilton Rating Scale for Depression.

Self-rated scales: GDS, CES-D, SDS (Zung), GRECC, BDI, POMS, 'Self-reported depressive symptoms', Chiribogas Positive/Negative Mood Scale, 'Depression': Messick's Differential Personality Inventory, Mood Scale, WDI, HADS, SDS, BSI, PRIME-MD. Observer-rated scales: HRSD/Ham-D, 'Staff rating of frequency of patient depression', SADS. Structural interviews: MADRS, The depressive symptoms measure, RDC.

increased use of adaptive- and approach-oriented coping strategies (as opposed to avoidance), acceptance, finding meaning, appraisal, positive reappraisal, and low avoidance coping at baseline were associated with less depression at follow-up. Both the longitudinal and crosssectional studies reported low levels of avoidance coping associated with lower levels of depressive symptoms. In addition, the 7 cross-sectional studies of higher quality reported low levels of passive coping and emotion-oriented coping (avoidance), denial, self-blame, complaint behavior, catastrophizing, and mystery beliefs to be associated with lower levels of depressive symptoms. Also, findings of high levels of problem- and task-oriented, active, cognitive and behavioral coping, ignoring pain, and coping self-statements were all associated with lower levels and absence of depressive symptoms. Three cross-sectional and 5 longitudinal studies reported of some additional aspects of strategies (i.e. avoidance, blaming, refocusing, praying, help seeking, appraisal and thought suppression) not being associated with symptoms of depression, or of symptoms of depression at follow-up (tables 2, 3). 
Table 5. Various instruments used in assessing coping

\begin{tabular}{|c|c|}
\hline Measurements & Number of studies \\
\hline Sense of coherence & Sense of coherence \\
\hline SOC (37) & $9(28,45,56,59,60,62,69,82,83)$ \\
\hline Control & Control \\
\hline Mastery (39) & $6(72,73,76,87,89,110)$ \\
\hline I/E Control of Reinforcement Scale (15) & $6(52,68,86,93,94,113)$ \\
\hline MHLC $(38,132)$ & $6(35,44,66,79,90,97)$ \\
\hline LOT (133) & $3(70,85,99)$ \\
\hline RLOC (134) & $2(47,92)$ \\
\hline Coping Self-Efficacy Scale (135) & $2(65,66)$ \\
\hline MHLC (136) & $1(81)$ \\
\hline The desired control questionnaire (137) & $1(112)$ \\
\hline Desired Control Measure (138) & $1(80)$ \\
\hline Sense of control over finances (18) & $1(95)$ \\
\hline CLOCM (139) & $1(104)$ \\
\hline HLOC (140) & $1(85)$ \\
\hline LOC(141) & $1(111)$ \\
\hline A structured measure of self-efficacy (142) & $1(75)$ \\
\hline $\operatorname{GSE}(143,144)$ & $1(85)$ \\
\hline IPQ-M (51) & $1(51)$ \\
\hline The Optimization in Primary and Secondary Control survey (145) & $1(50)$ \\
\hline Coping strategies & Coping strategies \\
\hline COPE $(146,147)$ & $4(44,49,61,108)$ \\
\hline WOC (21) & $3(70,71,84)$ \\
\hline CRI (148) & $2(48,91)$ \\
\hline CISS (149) & $2(101,114)$ \\
\hline CSQ (150) & $1(55)$ \\
\hline The Billings and Moos coping questionnaire (151) & $1(107)$ \\
\hline Indices of coping resources (152) & $1(79)$ \\
\hline HDLQ (122) & $1(105)$ \\
\hline CSQ (153) & $1(46)$ \\
\hline AAQ (22) & $1(77)$ \\
\hline PCI (154) & $1(106)$ \\
\hline SCSQ (23) & $1(43)$ \\
\hline UCL (155) & $1(104)$ \\
\hline CCQ (156) & $1(85)$ \\
\hline CERQ (157) & $1(114)$ \\
\hline Actions to cope (158) & $1(41)$ \\
\hline PTSD checklist (159) & $1(100)$ \\
\hline Coping resources (109) & $1(109)$ \\
\hline WBSI (160) & $1(57)$ \\
\hline SAM (161) & $1(71)$ \\
\hline HCA (162) & $1(99)$ \\
\hline Arthritis Self-Efficacy Scale $(163,164)$ & $1(78)$ \\
\hline CPCI (165) & $1(78)$ \\
\hline Pain Catastrophizing Scale (166) & $1(96)$ \\
\hline Vanderbilt Pain Management Inventory (167) & $1(46)$ \\
\hline Exercise coping measures (47) & $1(47)$ \\
\hline Revised coping questionnaire (no reference) & $1(103)$ \\
\hline Dispositional Resilience Scale II (168) & $1(53)$ \\
\hline Goal adjustment scale (169) & $1(98)$ \\
\hline Behavioral Coping Scale (170) & $1(96)$ \\
\hline
\end{tabular}


Table 5 (continued)

\begin{tabular}{ll}
\hline Measurements & Number of studies \\
\hline Religious coping & Religious coping \\
RCI $(25,171)$ & $5(25,26,35,58,74)$ \\
Intrinsic/Extrinsic Religiosity (I/E-R) Scale (172) & $2(35,86)$ \\
Brief RCOPE (173) & $1(26)$ \\
RPSS (34) & $1(35)$ \\
TSPC (174) & $1(36)$ \\
SIWB (175) & $1(36)$ \\
DSES (176) & $1(42)$ \\
\hline
\end{tabular}

Assessment of coping: SCSQ = The Simplified Coping Style Questionnaire; DSES = The Daily Spiritual Experiences Scale; I/E-R Scale = Intrinsic/Extrinsic Religiosity Scale; HLOC = Internal Health Locus of Control Scale; RPSS = Religious Problem-Solving Scales; RLOC = Recovery Locus of Control; RCI = Religious Coping Index; CSQ = Coping Strategies Questionnaires; $\mathrm{AAQ}=$ Acceptance and Action Questionnaire; PCI = The Proactive Coping Inventory; CRI = Coping Response Inventory; COPE = Coping Orientation to Problems Experienced Scale; CISS = Coping Inventory for Stressful Situations; MHLOC = Multidimensional Health Locus of Control Scale; HDLQ = Health and Daily Living Questionnaire; SPC = The Spiritual Practices Checklist; SIWB = The Spirituality Index of Well-Being; CPCI = The Chronic Pain Coping Inventory; HCA = Hearing Coping Assessment; LOT = Life Orientation Test; I/E Scale = The Rotter Internal and External Locus of Control Scale.

\section{Religious Coping}

From the total of 12 studies applying an instrument of religious coping, 6 were of higher quality ( 2 longitudinal and 4 cross-sectional; table 1) and all were recruiting older persons from clinical settings with participants suffering from depression (2 studies), somatic disorders, not specified (3 studies), and cancer (1 study; tables 2, 3). Among the longitudinal studies, high levels of positive religious coping and low levels of negative religious coping at baseline were related to lower levels of depressive symptoms at follow-up. Corresponding to the longitudinal studies, the cross-sectional studies found increased use of positive religious coping associated with lower levels of depressive symptoms. High levels of intrinsic religiosity were found to be important for a low depression score only in the cross-sectional studies. In 2 studies of higher quality ( 1 longitudinal and 1 cross-sectional), some additional aspects of religious coping (public and private religious practice, negative religious coping and religious coping index) were studied and shown not to be associated with depressive symptoms (tables 2,3).

\section{Discussion}

The discussion of the relation between coping and depression in older persons is based on the findings reported in the studies of higher quality. Although coping was defined in many ways throughout the studies, they formed four clusters of concepts, i.e. sense of coherence, control orientation, coping strategies, and religious coping. All high-quality studies reported findings of a relationship in which more adaptive coping (higher SOC, internal control orientation, active strategies, and use of more religious or positive coping) were associated with less depressive symptoms (tables 2, 3).

SOC may be viewed as a personal coping resource [32]. We found a positive relation between higher SOC and a lower degree of depressive symptoms in samples of older persons, 
which is in line with the findings from a systematic review on mixed age groups [29]. The finding that a stronger SOC is associated with fewer symptoms of depression indicates that the SOC may be a health-promoting factor, as proposed in the theory of salutogenesis, and that $\mathrm{SOC}$ as a coping resource contributes to the management of stress and promotes effective coping in older people [27].

Control orientation may be seen as another personal coping resource [32]. In this review of cross-sectional and longitudinal studies of higher quality, a strong and consistent relationship between higher internal control orientation and reduced levels of depressive symptoms were found in community-based populations among older persons with somatic disorders, and in older persons suffering from major depression or being dependent on care. This relation suggests that a high internal personal control orientation acts to prevent feelings of helplessness and depression, and may protect against these perceptions in times of stress and hardiness, as opposed to older persons having a low internal control orientation. This finding is in accordance with other research among mixed age groups [10,29] and older people $[7,65]$. Conclusively, the ability of an older person to retain good coping resources in terms of a strong SOC and high internal control seems important for mental health and in the understanding of depression in late life.

Regarding the association between coping strategies and depression, the majority of the high-quality studies, both cross-sectional and longitudinal, reported that emotion-oriented (avoidance) coping was positively related to more depressive symptoms and that more frequent use of problem-oriented (active strategies) coping was related to less depressive symptoms. The studies regarding coping strategies are community based or have recruited samples from clinical settings, and the results considering these samples indicate that active and problem-focused strategies may act as adaptive coping strategies in times of stress and protect against symptoms of depression. Studies including older persons suffering from major depression, cognitive decline, or dependence on care are scarce, but the results are in line with those from the community-based and clinical settings, including older persons suffering from somatic disorders. However, we found a great variability in the use of different instruments of coping strategies in all the samples. Many of these instruments were not well established or validated in this age group and it is difficult to compare the results with other studies (table 5).

Considering the associations between religious coping and depression, the main finding is of a significant relationship. Higher use of religious coping, in terms of more religious activities and religiosity as available coping resources, is associated with lower levels of depressive symptoms in the majority of the studies of high quality among both the cross-sectional and longitudinal studies. The studies examining religious coping and depression were mainly recruiting their samples from clinical settings. Religious practice is defined as the nature and time spent on religious activities, and could be understood in terms of coping strategies [26], while religious coping is a more broadly defined concept 'designed to assist people in the search for a variety of significant ends in stressful times: a sense of meaning and purpose, emotional comfort, personal control, intimacy with others, physical health, or spirituality' [33], with the latter definition expressing concepts more in line with resources of coping. Older persons suffering from somatic disorders and major depression may find meaning, emotional comfort and control in religious beliefs and activities, and religious coping could be a protective factor against depression in late life. The studies reviewed support this notion. Stronger and more personal religious beliefs were associated with lower depressive symptoms both at baseline and after a period of time. However, the number of studies is small and, further, the different concepts of religious coping might capture different phenomena (coping resources and coping strategies, respectively). 


\section{Strengths and Limitations of This Review}

It is a strength that we conducted a broad and thorough literature search, and that two researchers independently evaluated all the papers. We included all the coping perspectives used to study the relationship between coping and depression in this age group, in addition to evaluating all the papers according to quality criteria. The multitude of different concepts of coping, definitions and measures reported in the studies is an obvious limitation and may cause validity and reliability problems (table 5). We agree with other researchers who state that the variety of instruments for measuring coping and definitions of the concepts of coping make it difficult to compare the results of the studies and to draw firm conclusions, and we support the need for a further systematization of the theory and methodology of the coping field [63]. Few studies of older persons suffering from major depression and cognitive decline were found, and this also makes it difficult to conclude regarding coping and depression in these categories of elderly persons. Most of the studies including older persons suffering from major depression and cognitive decline have further excluded participants with more than a mild cognitive decline, so the included participants are not representative of the entire groups. This review is also limited because a meta-analytic approach in evaluating the studies statistically had to be omitted due to the variability in measures and designs. Also, because of the exclusion of articles written in languages other than English, the pool of research on this topic may have been limited. Consequently, the findings of this review must be interpreted cautiously.

\section{Implications for Clinical Practice}

The findings of this review imply that the instruments for measuring coping strategies and resources among older persons should be further theoretically and methodologically developed to reduce validity and reliability challenges. Despite the multitude of instruments of coping and the different settings where the studies took place, the results are quite unambiguous and show a significant relation between strategies and resources of coping and depressive symptoms in older persons, and the results are stable over time. There is also a need for validation and research on the instruments used for assessing strategies and resources of coping in samples of older persons with cognitive decline, including those who suffer from disorders like dementia and major depression. Instruments to assess resources and strategies of coping can be used to identify those at risk of developing a late-onset depressive disorder, a chronic course of recurrent depression, or worsening of depressive symptoms.

\section{Conclusion}

Our review of longitudinal and cross-sectional studies suggests a strong relationship between resources and strategies of coping and depressive symptoms in older persons from clinical and community settings. Higher SOC and internal control orientation and more use of active coping strategies and positive religious coping were related to lower levels of depressive symptoms. This finding supports the results from other reviews reporting a significant relation between concepts of coping and depressive symptoms. However, the huge variety of instruments measuring coping supports the need for a further systematization of the theory and the instruments of coping. In addition, further development of the instruments and research on coping in both populations of older persons suffering from major depression and cognitive decline is required. 
Bjørkløf et al.: Coping and Depression in Old Age: A Literature Review

\section{References}

- 1 Rosenvinge BH, Rosenvinge JH: Occurrence of depression in the elderly - a systematic review of 55 prevalence studies from 1990-2001 (in Norwegian). Tidsskr Nor Laegeforen 2003;123:928-929.

- 2 Seitz D, Purandare N, Conn D: Prevalence of psychiatric disorders among older adults in long-term care homes: a systematic review. Int Psychogeriatr 2010;22:1025-1039.

3 Mueller TI, Kohn R, Leventhal N, Leon AC, Solomon D, Coryell W, et al: The course of depression in elderly patients. Am J Geriatr Psychiatry 2004;12:22-29.

- 4 Barca ML, Selbaek G, Laks J, Engedal K: Factors associated with depression in Norwegian nursing homes. Int J Geriatr Psychiatry 2009;24:417-425.

- 5 Engedal K, Nordberg E, Moksnes KM, Henriksen HK, Bergem AL: Geriatric psychiatry - a specialty gaining recognition (in Norwegian). Tidsskr Nor Laegeforen 1997;117:3681-3683.

6 WHO: Global Burden of Disease - WHO2012 (updated 4.5.2012). http://www.nimh.nih.gov/statistics/pdf/ DALY-LeadingIndividualDisease.pdf.

7 Licht-Strunk E, van der Windt DA, van Marwijk HW, de Haan M, Beekman AT: The prognosis of depression in older patients in general practice and the community. A systematic review. Fam Pract 2007;24:168-180.

- 8 Mitchell AJ, Subramaniam H: Prognosis of depression in old age compared to middle age: a systematic review of comparative studies. Am J Psychiatry 2005;162:1588-1601.

- 9 Nilsson K, Gustafson L, Hultberg B: Survival in a large elderly population of patients with dementia and other forms of psychogeriatric diseases. Dement Geriatr Cogn Disord 2011;32:342-350.

10 Benassi VA, Sweeney PD, Dufour CL: Is there a relationship between locus of control orientation and depression? J Abnorm Psychol 1988;97:357-367.

11 Beekman AT, Deeg DJ, Geerlings SW, Schoevers RA, Smit JH, van Tilburg W: Emergence and persistence of late life depression: a 3-year follow-up of the Longitudinal Aging Study Amsterdam. J Affect Disord 2001;65:131138.

12 Lazarus RS, Lazarus BN: Coping with Aging. New York, Oxford University Press, 2006, pp 53-79.

13 Moos R: Psychological Techniques in the Assessment of Adaptive Behavior; Coelho GV, Hamburg DA, Adams JE (eds). New York, Basic Books, 1974.

14 Bandura A: Self-efficacy: toward a unifying theory of behavioral change. Psychol Rev 1977;84:191-215.

15 Rotter JB: Generalized expectancies for internal versus external control of reinforcement. Psychol Monogr 1966;80:1-28.

16 Thompson SC, Schlehofer MM: Perceived control; in Gerrard M, McCaul KD (eds): Health Behaviour Constructs: Theory, Measurement, and Research. dccps.cancer.gov/brp/constructs/perceived_control/pc5.html.

17 Craig AR, Franklin JA, Andrews G: A scale to measure locus of control of behaviour. Br J Med Psychol 1984;57: 173-180.

18 Lachman ME, Weaver SL: Sociodemographic variations in the sense of control by domain: findings from the MacArthur studies of midlife. Psychol Aging 1998;13:553-562.

19 Folkman S, Lazarus RS: An analysis of coping in a middle-aged community sample. J Health Soc Behav 1980; 21:219-239.

20 Folkman S, Lazarus RS, Gruen RJ, DeLongis A: Appraisal, coping, health status, and psychological symptoms. J Pers Soc Psychol 1986;50:571-579.

21 Folkman S, Lazarus RS: Manual for the Ways of Coping Questionnaire. Palo Alto, Consulting Psychologist Press, 1988.

22 Hayes SC, Strosahl K, Wilson KG, Bisset RT, Pistorello J, Taormino D: Measuring experiential avoidance: a preliminary test of a working model. Psychol Rec 2004;54:553-578.

23 Xie YN: The Simplified Coping Style Questionnaire. Chin J Psychol 1995;7:123-124.

24 Pargament KI: The Psychology of Religion and Coping - Theory, Research, Practice. New York, Guilford Press, 1997.

25 Koenig HG, Cohen HJ, Blazer DG, Pieper C, Meador KG, Shelp F, et al: Religious coping and depression among elderly, hospitalized medically ill men. Am J Psychiatry 1992;149:1693-1700.

26 Bosworth HB, Park KS, McQuoid DR, Hays JC, Steffens DC: The impact of religious practice and religious coping on geriatric depression. Int J Geriatr Psychiatry 2003;18:905-914.

27 Antonovsky A: Health, Stress and Coping. San Fransisco, Jossey-Bass, 1979.

28 Rennemark M, Hagberg B: What makes old people perceive symptoms of illness? The impact of psychological and social factors. Aging Ment Health 1999;3:79-87.

29 Eriksson M, Lindstrom B: Antonovsky's sense of coherence scale and the relation with health: a systematic review. J Epidemiol Community Health 2006;60:376-381.

30 Oxman AD: Systematic reviews: checklists for review articles. BMJ 1994;309:648-651.

31 Radloff LS: The CES-D Scale. Appl Psycholog Meas 1977;1:385-401.

32 Wheaton B: Models for the stress-buffering functions of coping resources. J Health Soc Behav 1985;26:352364.

-33 Pargament KI, Smith BW, Koenig HG, Perez L: Patterns of positive and negative religious coping with major life stressors. J Sci Study Relig 1998;37:710-724.

34 Pargament KI, Kennell J, Hathaway W: Religion and the problem-solving process; three styles of coping. J Sci Study Relig 1988;27:90-104. 
Bjørkløf et al.: Coping and Depression in Old Age: A Literature Review

35 Grosse-Holtforth M, Pathak A, Koenig HG, Cohen HJ, Pieper CF, Vanhook LG: Medical illness, religion, health control and depression of institutionalized medically ill veterans in long-term care. Int J Geriatr Psychiatry 1996;11:613-620.

-36 Whelan-Gales MA, Quinn Griffin MT, Maloni J, Fitzpatrick JJ: Spiritual well-being, spiritual practices, and depressive symptoms among elderly patients hospitalized with acute heart failure. Geriatr Nurs 2009;30: 312-317.

37 Antonovsky A: The salutogenic model as a theory to guide health promotion. Health Promot Int 1996;11: 11-18.

38 Wallston KA, Wallston BS, DeVellis R: Development of the Multidimensional Health Locus of Control (MHLC) Scales. Health Educ Monogr 197;6:160-170.

39 Pearlin LI, Schooler C: The structure of coping. J Health Soc Behav 1978;19:2-21.

40 Harris T, Cook DG, Victor C, DeWilde S, Beighton C: Onset and persistence of depression in older people results from a 2-year community follow-up study. Age Ageing 2006;35:25-32.

-41 Cuijpers P, Steunenberg B, Van Straten A: Actions taken to cope with depressed mood: the role of personality traits. Aging Ment Health 2007;11:457-463.

42 Han J, Richardson VE: The relationship between depression and loneliness among homebound older persons: does spirituality moderate this relationship? J Relig Spiritual Soc Work 2010;29:218-236.

43 Xie L-Q, Zhang J-P, Peng F, Jiao N-N: Prevalence and related influencing factors of depressive symptoms for empty-nest elderly living in the rural area of YongZhou, China. Arch Gerontol Geriatr 2008;50:24-29.

44 Paukert AL, LeMaire A, Cully JA: Predictors of depressive symptoms in older veterans with heart failure. Aging Ment Health 2009;13:601-610.

45 Schneider G, Driesch G, Kruse A, Wachter M, Nehen HG, Heuft G: What influences self-perception of health in the elderly? The role of objective health condition, subjective well-being and sense of coherence. Arch Gerontol Geriatr 2004;39:227-237.

46 Lopez-Lopez A, Montorio I, Izal M, Velasco L: The role of psychological variables in explaining depression in older people with chronic pain. Aging Ment Health 2008;12:735-745.

$\checkmark 47$ Johnston M, Morrison V, Macwalter R, Partridge C: Perceived control, coping and recovery from disability following stroke. Psychol Health 1999;14:181-192.

48 Croyle KL, Tröster AI, Fields JA, Straits-Tröster KA, Lyons KE, Pahwa R: Presurgical coping, depression, and quality of life in persons with Parkinson's Disease. J Clin Psychol Med Settings 2003;10:101-107.

49 Klein DM, Turvey CL, Pies CJ: Relationship of coping styles with quality of life and depressive symptoms in older heart failure patients. J Aging Health 2007;19:22-38.

50 Wallace ML, Dombrovski AY, Morse JQ, Houck PR, Frank E, Alexopoulos GS, et al: Coping with health stresses and remission from late-life depression in primary care: a two-year prospective study. Int J Geriatr Psychiatry 2012;27:178-186.

51 Hurt CS, Burns A, Brown RG, Barrowclough C: Why don't older adults with subjective memory complaints seek help? Int J Geriatr Psychiatry 2012;27:394-400.

-52 Anderson-Hanley C, Meshberg SR, Marsh MA: The effects of a control-enhancing intervention for nursing home residents: cognition and locus of control as moderators. Palliat Support Care 2003;1:111-120.

-53 Engel JH, Siewerdt F, Jackson R, Akobundu U, Wait C, Sahyoun N: Hardiness, depression, and emotional wellbeing and their association with appetite in older adults. J Am Geriatr Soc 2011;59:482-487.

54 Folstein MF, Folstein SE, McHugh P: 'Mini-Mental State'. A practical method for grading the cognitive state of patients for the clinician. J Psychiatr Res 1975;12:189-198.

55 Lynch TR, Morse JQ, Mendelson T, Robins CJ: Dialectical behavior therapy for depressed older adults: a randomized pilot study. Am J Geriatr Psychiatry 2003;11:33-45.

56 Wiktorsson S, Marlow T, Runeson B, Skoog I, Waern M: Prospective cohort study of suicide attempters aged 70 and above: one-year outcomes. J Affect Disord 2011;134:333-340.

-57 Rosenthal MZ, Cheavens JS, Compton JS, Thorp SR, Lynch TR: Thought suppression and treatment outcome in late-life depression. Aging Ment Health 2005;9:35-39.

58 Payman V, George K, Ryburn B: Religiosity of depressed elderly inpatients. Int J Geriatr Psychiatry 2007;23: $16-21$.

59 Ziv N, Roitman DM, Katz N: Problem solving, sense of coherence and instrumental ADL of elderly people with depression and normal control group. Occup Ther Int 1999;6:243.

60 Mellqvist M, Wiktorsson S, Joas E, Ostling S, Skoog I, Waern M: Sense of coherence in elderly suicide attempters: the impact of social and health-related factors. Int Psychogeriatr 2011;1:1-8.

61 Fisher BM, Segal DL, Coolidge FL: Assessment of coping in cognitively impaired older adults: a preliminary study. Clin Gerontol 2003;26:3-12.

62 Drageset J, Espehaug B, Kirkevold M: The impact of depression and sense of coherence on emotional and social loneliness among nursing home residents without cognitive impairment - a questionnaire survey. J Clin Nurs 2012;21:965-974.

63 Skinner EA, Edge K, Altman J, Sherwood H: Searching for the structure of coping: a review and critique of category systems for classifying ways of coping. Psychol Bull 2003;129:216-269.

64 Krause N: Stress and coping: reconceptualizing the role of locus of control beliefs. J Gerontol 1986;41:617622. 
Bjørkløf et al.: Coping and Depression in Old Age: A Literature Review

65 Cole MG, Dendukuri N: Risk factors for depression among elderly community subjects: a systematic review and meta-analysis. Am J Psychiatry 2003;160:1147-56.

66 Kraaij V, Garnefski N, Maes S: The joint effects of stress, coping, and coping resources on depressive symptoms in the elderly. Anxiety Stress Coping 2002;15:163-177.

67 Singh NA, Stavrinos TM, Scarbek Y, Galambos G, Liber C, Fiatarone Singh MA: A randomized controlled trial of high versus low intensity weight training versus general practitioner care for clinical depression in older adults. J Gerontol A Biol Sci Med Sci 2005;60:768-776.

68 Krause N: Chronic financial strain, social support, and depressive symptoms among older adults. Psychol Aging 1987;2:185-192.

69 Zielinska-Wieczkowska H, Ciemnoczolowski W, Kedziora-Kornatowska K, Muszalik M: The sense of coherence (SOC) as an important determinant of life satisfaction, based on own research, and exemplified by the students of University of the Third Age (U3A). Arch Gerontol Geriatr 2012;54:238-241.

-70 King RB, Shade-Zeldow Y, Carlson CE, Feldman JL, Philip M: Adaptation to stroke: a longitudinal study of depressive symptoms, physical health, and coping process. Top Stroke Rehabil 2002;9:46-66.

-71 Rochette A, Bravo G, Desrosiers J, St-Cyr Tribble D, Bourget A: Adaptation process, participation and depression over six months in first-stroke individuals and spouses. Clin Rehabil 2007;21:554-562.

72 Cohen CI, Goh KH, Yaffee RA: Depression outcome among a biracial sample of depressed urban elders. Am J Geriatr Psychiatry 2009;17:943-952.

73 Zarit SH, Femia EE, Gatz M, Johansson B: Prevalence, incidence and correlates of depression in the oldest old: the OCTO study. Aging Ment Health 1999;3:119-128.

74 Koenig HG, Cohen HJ, Blazer DG, Kudler HS, Krishnan KR, Sibert TE: Religious coping and cognitive symptoms of depression in elderly medical patients. Psychosomatics 1995;36:369-375.

75 Chou KL, Chi I: Stressful life events and depressive symptoms: social support and sense of control as mediators or moderators? Int J Aging Hum Dev 2001;52:155-171.

76 Jang Y, Chiriboga DA, Kim G, Rhew S: Perceived discrimination in older Korean Americans. Asian Am J Psychol 2010;1:129-135.

77 Andrew DH, Dulin PL: The relationship between self-reported health and mental health problems among older adults in New Zealand: experiential avoidance as a moderator. Aging Ment Health 2007;11:596-603.

78 Turner JA, Ersek M, Kemp C: Self-efficacy for managing pain is associated with disability, depression, and pain coping among retirement community residents with chronic pain. J Pain 2005;6:471-479.

-79 Unger DG, Jacobs SB: Couples and chronic obstructive airway diseases: the role of gender in coping and depression. Womens Health 1995;1:237-255.

80 Windsor TD, Anstey KJ, Butterworth P, Luszcz MA, Andrews GR: The role of perceived control in explaining depressive symptoms associated with driving cessation in a longitudinal study. Gerontologist 2007;47:215223.

81 Molinary V, Niederehe G: Locus of control, depression, and anxiety in young and old adults: a comparison study. Int J Aging Hum Dev 1984;20:1984-1985.

82 Graham K: Alcohol abstention among older adults: reasons for abstaining and characteristics of abstainers. Addict Res 1998;6:473-487.

-83 Takkinen S, Ruoppila I: Meaning in life as an important component of functioning in old age. Int J Aging Hum Dev 2001;53:211-231.

84 Hurt CS, Burns A, Barrowclough C: Perceptions of memory problems are more important in predicting distress in older adults with subjective memory complaints than coping strategies. Int Psychogeriatr 2011;23:13341343.

-85 Schroder KE: Coping competence as predictor and moderator of depression among chronic disease patients. J Behav Med 2004;27:123-145.

-86 Fehring RJ, Miller JF, Shaw C: Spiritual well-being, religiosity, hope, depression, and other mood states in elderly people coping with cancer. Oncol Nurs Forum 1997;24:663-671.

87 Van den Heuvel N, Smits CH, Deeg DJ, Beekman AT: Personality: a moderator of the relation between cognitive functioning and depression in adults aged 55-85. J Affect Disord 1996;41:229-240.

88 Harris T, Cook DG, Victor C, Rink E, Mann AH, Shah S, et al: Predictors of depressive symptoms in older people - a survey of two general practice populations. Age Ageing 2003;32:510-518.

-89 Zunzunegui MV, Beland F, Llacer A, Leon V: Gender differences in depressive symptoms among Spanish elderly. Soc Psychiatry Psychiatr Epidemiol 1998;33:195-205.

$\$ 90$ Wallhagen MI, Strawbridge WJ, Kaplan GA, Cohen RD: Impact of internal health locus of control on health outcomes for older men and women: a longitudinal perspective. Gerontologist 1994;34:299-306.

-91 Holahan CJ, Moos RH, Holahan CK, Brennan PL: Social support, coping, and depressive symptoms in a late-middle-aged sample of patients reporting cardiac illness. Health Psychol 1995;14:152-163.

$\$ 92$ Thomas SA, Lincoln NB: Factors relating to depression after stroke. Br J Clin Psychol 2006;45:49-61.

$\$ 93$ Byrd M: Letting the inmates run the asylum: the effects of control and choice on the institutional lives of older adults. Act Adapt Aging 1983;3:3-11.

-94 Hanes CR, Wild BS: Locus of control and depression among noninstitutionalized elderly persons. Psychol Rep 1977;41:581-582.

95 Chou K-L, Chi I: Financial strain and depressive symptoms in Hong Kong elderly Chinese: the moderating or mediating effect of sense of control. Aging Ment Health 2000;5:23-30. 
Bjørkløf et al.: Coping and Depression in Old Age: A Literature Review

96 Hawker GA, Gignac MA, Badley E, Davis AM, French MR, Li Y, et al: A longitudinal study to explain the paindepression link in older adults with osteoarthritis. Arthritis Care Res (Hoboken) 2011;63:1382-1390.

97 Abu-Bader SH, Tirmazi MT, Ross-Sheriff F: The impact of acculturation on depression among older Muslim immigrants in the United States. J Gerontol Soc Work 2011;54:425-448.

98 Schuz B, Wurm S, Schollgen I, Tesch-Romer C: What do people include when they self-rate their health? Differential associations according to health status in community-dwelling older adults. Qual Life Res 2011;20: 1573-1580.

99 Andersson G, Melin L, Lindberg P, Scott B: Dispositional optimism, dysphoria, health, and coping with hearing impairment in elderly adults. Audiology 1995;34:76-84.

100 Dulin PL, Passmore T: Avoidance of potentially traumatic stimuli mediates the relationship between accumulated lifetime trauma and late-life depression and anxiety. J Trauma Stress 2010;23:296-299.

101 Pokorski M, Warzecha A: Depression and religiosity in older age. Eur J Med Res 2011;16:401-406.

102 Black SA, Markides KS, Miller TQ: Correlates of depressive symptomatology among older community-dwelling Mexican Americans: the Hispanic EPESE. J Gerontol B Psychol Sci Soc Sci 1998;53:S198-S208.

103 Price A, Goodwin L, Rayner L, Shaw E, Hansford P, Sykes N, et al: Illness perceptions, adjustment to illness, and depression in a palliative care population. J Pain Symptom Manage 2012;43:819-832.

104 Derks W, de Leeuw JRJ, Hordijk GJ, Winnubst JAM: Differences in coping style and locus of control between older and younger patients with head and neck cancer. Clin Otolaryngol 2005;30:186-192.

105 Foster JM, Gallagher D: An exploratory study comparing depressed and nondepressed elders' coping strategies. J Gerontol 1986;41:91-93.

106 Greenglass E, Fiksenbaum L, Eaton J: The relationship between coping, social support, functional disability and depression in the elderly. Anxiety Stress Coping 2006;19:15-31.

107 Ehmann TS, Beninger RJ, Gawel MJ, Riopelle RJ: Coping, social support, and depressive symptoms in Parkinson's disease. J Geriatr Psychiatry Neurol 1990;3:85-90.

108 Catanzaro SJ, Horaney F, Creasey G: Hassles, coping, and depressive symptoms in an elderly community sample: the role of mood regulation expectancies. J Counsel Psychol 1995;42:259-265.

109 Mui AC, Burnette D: Coping resources and self-reported depressive symptoms among frail older ethnic women. J Soc Serv Res 1996;21:19-36.

110 Pouwer F, Beekman AT, Nijpels G, Dekker JM, Snoek FJ, Kostense PJ, et al: Rates and risks for co-morbid depression in patients with type 2 diabetes mellitus: results from a community-based study. Diabetologia 2003;46:892-898.

111 Arnetz BB: Gerontic occupational therapy - psychological and social predictors of participation and therapeutic benefits. Am J Occup Ther 1985;39:460-465.

112 Ziegler M, Reid DW: Correlates of locus of desired control in two samples of elderly persons: community residents and hospitalized patients. J Consult Clin Psychol 1979;47:977-979.

113 Krause N: Chronic strain, locus of control, and distress in older adults. Psychol Aging 1987;2:375-382.

114 Kraaij V, Pruymboom E, Garnefski N: Cognitive coping and depressive symptoms in the elderly: a longitudinal study. Aging Ment Health 2002;6:275-281.

115 Yesavage JA, Brink TL, Rose TL, Lum O, Huang V, Adey M, et al: Development and validation of a geriatric depression screening scale: a preliminary report. J Psychiatr Res 1982;17:37-49.

116 Sheikh JI, Yesavage JA: A knowledge assessment test for geriatric psychiatry. Hosp Community Psychiatry 1985;36:1160-1166.

117 Beck AT, Ward CH, Mendelson M, Mock J, Erbaugh J: An inventory for measuring depression. Arch Gen Psychiatry 1961;4:561-571.

118 Miller IW, Bishop S, Norman WH, Maddever H: The Modified Hamilton Rating Scale for Depression: reliability and validity. Psychiatry Res 1985;14:131-142.

119 Zung WW: A self-rating depression scale. Arch Gen Psychiatry 1965;12:63-70.

120 McNair DM, Lorr M, Droppleman LF: Profile of Mood States. San Diego, EdITS, 1971.

121 Derogatis LR: Brief Symptom Inventory: Administration, Scoring and Procedures Manual. Minneapolis, National Computer Systems, 1993.

122 Moos RH, Cronkite RC, Billings AG, Finney JW: Health and Daily Living Form Manual. Standford, SUSoM, 1983.

123 Montgomery SA, Asberg M: A new depression scale designed to be sensitive to change. Br J Psychiatry 1979; 134:382-389.

124 Zigmond AS, Snaith RP: The hospital anxiety and depression scale. Acta Psychiatr Scand 1983;67:361-370.

125 Kaplan GA, Roberts RE, Camacho TC, Coyne JC: Psychosocial predictors of depression. Prospective evidence from the human population laboratory studies. Am J Epidemiol 1987;125:206-220.

126 Koenig HG, Cohen HJ, Blazer DG, Meador KG, Westlund R: A brief depression scale for use in the medically ill. Int J Psychiatry Med 1992;22:183-195.

127 Spitzer RL, Endicott J, Robins E: Research diagnostic criteria: rationale and reliability. Arch Gen Psychiatry 1978;35:773-782.

128 Snaith RP, Ahmed SN, Mehta S, Hamilton M: Assessment of the severity of primary depressive illness. Wakefield self-assessment depression inventory. Psychol Med 1971;1:143-149.

129 Endicott J, Spitzer RL: A diagnostic interview: the schedule for affective disorders and schizophrenia. Arch Gen Psychiatry 1978;35:837-844. 
Bjørkløf et al.: Coping and Depression in Old Age: A Literature Review

130 Spitzer R, Kroenke K, Williams J: Validation and utility of a self-report version of PRIME-MD: the PHQ primary care study. JAMA 1999;282:1737-1744.

131 Johnston A, Benton CP, Morgan MJ: Concurrent measurement of perceived speed and speed discrimination threshold using the method of single stimuli. Vision Res 1999;39:3849-3854.

132 Wallston BS, Wallston KA, Kaplan GD, Maides SA: Development and validation of the health locus of control (HLC) scale. J Consult Clin Psychol 1976;44:580-585.

133 Scheier MF, Carver CS: Optimism, coping, and health: assessment and implications of generalized outcome expectancies. Health Psychol 1985;4:219-247.

134 Johnston M, Wright, S, Weinman J: Measures in Health Psychology: A User's Portfolio. Windsor, NFER-Nelson, 1995.

135 Sherer M, et al: The Self-efficacy Scale: construction and validation. Psychol Rep 1982;51:663-671.

136 Levenson H: Distinctions within the concept of internal-external control: development of a new scale. 80th Annual Convention of the American Psychological Association, Honolulu, 1972, pp 261-262.

137 Reid DW, Ziegler MA: A survey of the reinforcements and activities elderly citizens feel are important for their general happiness. Essence 1977;2:5-24.

138 Luszcz MA, Bryan J, Kent P: Predicting episodic memory performance of very old men and women: contributions from age, depression, activity, cognitive ability, and speed. Psychol Aging 1997;12:340-351.

139 Pruyn JF, Borne HW, Reuver RS: The Locus of Control scale for cancer patients. T Soc Gezondsheidsz 1988;66: 404-408.

140 Ferring D, Filipp S-H: Der Fragebogen zur Erfassung gesundheitsbezogener Kontrollueberzeugungen. Kurzbericht. Z Klinische Psychol 1989;18:285-289.

141 Reid DW, Haas G, Hawkings D: Locus of desired control and positive self-concept of the elderly. J Gerontol 1977;32:441-450.

142 McAvay GJ, Seeman TE, Rodin J: A longitudinal study of change in domain-specific self-efficacy among older adults. J Gerontol B Psychol Sci Soc Sci 1996;51:P243-P253.

143 Schwarzer R, Baessler J, Kwiatek P, Schroder KEE, Zhang JX: The assessment of optimistic self-beliefs: comparison of the German, Spanish, and Chinese versions of the General Self-Efficacy Scale. Appl Psychol Int Rev 1997;46:69-88.

144 Schwarzer R, Jerusalem M: Generalized Self-Efficacy Scale. 2001 (cited November 1, 2001). http://userpage. fu-berlin.de/ health/selfscal.htm.

145 Schulz R, Heckhausen J: Health-Specific Optimization in Primary and Secondary Control Scales. Pittsburg, University of Pittsburg, 1998.

146 Carver CS: You want to measure coping but your protocol's too long: consider the brief COPE. Int J Behav Med 1997; 4:92-100.

147 Carver CS, Scheier MF, Weintraub JK: Assessing coping strategies: a theoretically based approach. J Pers Soc Psychol 1989;56:267-283.

148 Moos RH: Coping Responses Inventory: Adult Form Manual. Odessa, Psychological Assessment Resources, 1993.

149 Endler NS, Parker JD: Multidimensional assessment of coping: a critical evaluation. J Pers Soc Psychol 1990; 58:844-854.

150 Roger D, Jarvis G, Najarian B: Detachment and coping: the construction and validation of a new scale for measuring coping strategies. Pers Individ Dif 1993;15:619-626.

151 Billings AG, Moos RH: The role of coping responses and social resources in attenuating the stress of life events. J Behav Med 1981;4:139-157.

152 Moos RH, Cronkite RC, Billings AG, Finney JW: Health and Daily Living Form Manual. Stanford, Department of Psychiatry Stanford University, 1987.

153 Rosenstiel AK, Keefe FJ: The use of coping strategies in chronic low back pain patients: relationship to patient characteristics and current adjustment. Pain 1983;17:33-44.

154 Greenglass ER, Schwarzer R, Taubert S: The Proactive Coping Inventory: A Multidimensional Research Instrument. 1999. www.psych.yorku.ca/greenglass.

155 Schreurs KM, van de Willige G, Brosschot JF: De Utrechtse Coping Lijst. Lisse, Swets and Zeitlinger, 1993.

156 Schroder KE: Self-Regulation Competence in Coping with Chronic Disease. Berlin, Waxman, 1997.

157 Garnefski N, Kraaij V, Spinhoven PH: Negative life events, cognitive emotion regulation, and emotional problems. Pers Individ Dif 2001;30:1311-1327.

158 Jorm AF, Griffiths KM, Christensen H, Parslow RA, Rogers B: Actions taken to cope with depression at different levels of severity: a community survey. Psychol Med 2004;34:293-299.

159 Schinka JA, Brown LM, Borenstein AR, Mortimer JA: Confirmatory factor analysis of the PTSD checklist in the elderly. J Trauma Stress 2007;20:281-289.

160 Wegner DM, Zanakos S: Chronic thought suppression. J Pers 1994;62:616-640.

161 Peacock EJ, Wong PT: The stress appraisal measure: a multidimensional approach to cognitive appraisal. Stress Med 1990;6:227-236.

162 Andersson G, Melin L, Lindberg P, Scott B: Development of a short scale for self-assessment of experiences of hearing impairment: the hearing coping assessment. Scand Audiol 1995;24:147-154.

163 Gonzalez VM, Stewart A, Ritter PL, Lorig K: Translation and validation of arthritis outcome measures into Spanish. Arthritis Rheum 1995;38:1429-1446. 
164 Lorig K, Stewart A, Ritter PL, Gonzalez VM, Laurent D, Lynch J: Outcome Measures for Health Education and Other Health Care Interventions. Thousand Oaks, Sage Publications, 1996.

165 Jensen MP, Turner JA, Romano JM, Strom SE: The chronic pain coping inventory: development and preliminary validation. Pain 1995;60:203-216.

166 Sullivan MJ, Bishop SR, Pivik J: The Pain Catastrophizing Scale: development and validation. Psychol Assess 1995; 7:524-532.

167 Brown GK, Nicassio PM: Development of a questionnaire for the assessment of active and passive coping strategies in chronic pain patients. Pain 1987;31:53-64.

168 Sinclair R, Oliver C, Ippolito J: Development and validation of a short measure of hardiness. Portland, Portland State University Department of Psychology, 2003.

169 Brandtstadter J, Renner G: Tenacious goal pursuit and flexible goal adjustment: explication and age-related analysis of assimilative and accommodative strategies of coping. Psychol Aging 1990;5:58-67.

170 Gignac MA, Cott C, Badley EM: Adaptation to chronic illness and disability and its relationship to perceptions of independence and dependence. J Gerontol B Psychol Sci Soc Sci 2000;55:362-372.

171 Koenig HG: Differences in psychosocial and health correlates of major and minor depression in medically ill older adults. J Am Geriatr Soc 1997;45:1487-1495.

172 Gorsuch R, McPherson S: Intrinsic/extrinsic measurement: I/E-revised and single-item scales. J Sci Study Relig 1989;28:348-354.

173 FINioaw Group: Multidimensional measurement of religiousness/spirituality for use in health research. A report of national working group supported by the Fetzer Institute in collaboration with the National Institute on Aging. Kalamazoo, Fetzer Institute, 1999.

174 Bussing A, Mattiessen PF, Ostermann T: Engagement of patients in religious and spiritual practices. Health Qual Life Outcomes 2005;3:53-63.

175 Daaleman TP, Frey BB: The Spirituality Index of Well-Being: a new instrument for health-related quality-oflife research. Ann Fam Med 2004;2:499-503.

176 Underwood LG, Teresi JA: The Daily Spiritual Experience Scale: development, theoretical description, reliability, exploratory factor analysis, and preliminary construct validity using health-related data. Ann Behav Med 2002;24:22-33. 